\title{
The Vibrio cholerae ToxR/TcpP/ToxT virulence cascade: distinct roles for two membrane-localized transcriptional activators on a single promoter
}

\author{
Eric S. Krukonis, ${ }^{1}$ Rosa R. Yu ${ }^{2}$ and Victor J. DiRita ${ }^{1,2 *}$ \\ ${ }^{1}$ Unit for Laboratory Animal Medicine, and \\ ${ }^{2}$ Department of Microbiology and Immunology, \\ University of Michigan Medical School, Ann Arbor, \\ MI 48109-0614, USA.
}

\section{Summary}

ToxR is required in Vibrio cholerae for transcriptional activation of the toxT gene, the protein product of which activates numerous genes involved in virulence. Although ToxR cannot activate the toxT promoter in Escherichia coli, the products of the tcpPH operon are shown here to activate the toxT promoter, and co-expression with ToxRS enhances activation. An identical pattern was seen in a $\Delta t c p P \Delta$ toxR strain of $V$. cholerae when TcpPH or ToxRS was expressed from plasmids. Although overexpression of the TcpP/H proteins in $V$. cholerae partially complemented both a $\Delta$ toxR strain and a $\Delta t c p P \Delta$ tox $R$ double mutant for toxin production and toxT-lacZ activation, the presence of ToxR greatly increased their expression. Analysis of a toxT-lacZ promoter deletion series demonstrated that TcpP was able to interact functionally with the toxT promoter downstream of the ToxR binding site. This was confirmed using electrophoretic mobility shift assays of this toxT promoter deletion series and DNase I footprinting analysis, which showed that TcpP interacts with the promoter region from -51 to -32 , whereas Tox $R$ protected a region from -100 to -69 . In addition, membranes containing endogenous levels of ToxR bound more readily to the toxT promoter than did membranes containing only TcpP. Characterization of a number of tcpP substitution mutants revealed one derivative (TcpP-H93L) that, when overexpressed, was markedly defective for toxT activation, cholera toxin and TcpA (toxin co-regulated pilus) production and DNA binding; however, toxT activation by TcPP-H93L was restored in the presence of ToxR, suggesting that Tox $R$ can provide the promoter recognition function for toxT activation. Two additional mutant derivatives, TcpP-W68L and TcpP-R86A, failed to activate toxT or

Accepted 14 July, 2000. *For correspondence. E-mail vdirita @umich. edu; Tel. (+1) 734936 3804; Fax (+1) 7349363235. direct toxin and TcpA production in the presence or absence of ToxR. Both TcpP-W68L and TcpP-R86A, like TcpP-H93L, were defective for DNA binding. Finally, a ToxR mutant derivative, ToxR-G80S, served to separate the different roles of ToxR on different promoters. Although ToxR-G80S was inefficient at activating the ompU promoter in $V$. cholerae (ompU encodes an outer membrane porin regulated by ToxR), it was fully capable of activating the toxT promoter. These data suggest that ToxR is not a direct activator in the toxT expression system but, instead, enhances the activity of TcpP, perhaps by recruiting it to the toxT promoter under conditions in which expression levels of TcpP are too low for it to activate toxT efficiently on its own.

\section{Introduction}

Vibrio cholerae is the causative agent of the diarrhoeal disease cholera. Secretion of cholera toxin by the bacterium leads to elevated cAMP levels in the cells lining the host intestinal tract, resulting in voluminous secretion of water and electrolytes. Although cholera toxin is the effector of these changes in host physiology, a number of other factors are known to be critical for establishing a productive infection. These include the regulatory proteins ToxR and ToxT (Miller et al., 1987; DiRita et al., 1991), the colonization factor toxin coregulated pilus (TCP, encoded by tcpA; Taylor et al., 1987), several accessory colonization factors (AcfA-D; Peterson and Mekalanos, 1988) and the recently identified response regulator homologue varA (Wong et al., 1998). ToxR and ToxT operate in a cascade-like fashion in which ToxR activates the tox $T$ promoter, and ToxT acts downstream to regulate various promoters, including $\operatorname{ctx} A$, tcpA, tcpC, tcpl and acfA (DiRita et al., 1991). Mutations in toxR result in loss of production of all these factors as well as the ToxT-independent product OmpU (Miller and Mekalanos, 1988), an outer membrane porin (Chakrabarti et al., 1996), whereas mutations in toxT prevent production of all these factors with the exception of OmpU (Champion et al., 1997). Furthermore, heterologous expression of ToxT can bypass the effects of a tox $R$ mutation, except with respect to OmpU (Champion et al., 1997).

ToxR appears to have gained control of virulence gene 
expression via ToxT after an evolutionary event in which the $t c p$-acf gene cluster (the Vibrio pathogenicity island, VPI), which includes toxT, was delivered to $V$. cholerae by horizontal transfer via the newly described VPI phage (Kovach et al., 1996; Karaolis et al., 1998; 1999). Similarly, the cholera toxin locus is also on a mobile genetic element, the CTX phage (Waldor and Mekalanos, 1996). Thus, both the major virulence determinant of $V$. cholerae, cholera toxin, and its regulator, ToxT, were obtained by independent horizontal transfer events.

Recently, a second level of regulation was shown to impinge on toxT expression. Mutations in the tcpPH locus lying upstream of the TCP biosynthetic operon led to loss of toxT transcription (Carroll et al., 1997; Häse and Mekalanos, 1998; Yu and DiRita, 1999). Additionally, overexpression of the TcpP protein activates tox $T$ transcription, as measured by both expression of a toxT-lac $Z$ gene fusion and primer extension analysis in $V$. cholerae (Häse and Mekalanos, 1998; Yu and DiRita, 1999). Thus, transcription of the major regulator of virulence factor transcription, toxT, is dependent on the activities of two transcription factors, ToxR and TcpP.

ToxR and TcpP are homologous to the OmpR family of transcriptional activators. Unlike many OmpR family activators, both proteins contain DNA-binding motifs in their $\mathrm{N}$-termini rather than in their C-termini (Miller et al., 1987; Ogierman et al., 1996), and both are predicted to reside within the inner membrane of the bacterium with substantial periplasmic domains: 96 (ToxR) and 52-60 (depending on the location of the putative transmembrane domain of TcpP) amino acids (Miller et al., 1987; DiRita and Mekalanos, 1991; Ogierman et al., 1996). In addition, both toxR and tcpP are encoded in polycistronic operons with downstream genes (toxS and $t c p H$ respectively) encoding effector proteins that enhance the activity of their cognate partners (Carroll et al., 1997; Yu and DiRita, 1999; E. S. Krukonis, V. J. DiRita and B. Hammer unpublished observations).

Transcription of $t c p P H$ has been shown to be regulated by both temperature and pH (Thomas et al., 1995; Carroll et al., 1997), raising the possibility that environmental regulation of the ToxR/TcpP/ToxT cascade in $V$. cholerae manifests itself at the level of TcpP production. Transcription of $t c p P H$ has recently been shown to be controlled by two additional factors, AphA and AphB (Kovacikova and Skorupski, 1999; Skorupski and Taylor, 1999).

In this study, we undertook a mechanistic analysis of toxT transcriptional regulation by ToxR and TcpP. We show that, as predicted from previous studies demonstrating the inability of ToxR alone to activate toxT (Higgins and DiRita, 1994), TcpP functions with ToxR for toxT activation, consistent with what has been reported recently by others (Häse and Mekalanos, 1998; Murley et al., 1999). We expand this observation to show that TcpP binds to the
toxT promoter at a position downstream of the ToxR binding site, closer to the basal elements of the promoter. We present a model for the interaction of ToxR and TcpP with the toxT promoter, in which ToxR facilitates the ability of TcpP to interact with RNA polymerase, leading to transcriptional activation of tox $T$ and subsequent coordinate activation of virulence gene expression.

\section{Results}

TcpP can activate a toxT-lacZ fusion in E. coli, but not ompU-lacZ or ctxA-lacZ

Previous studies in Escherichia coli demonstrated that ToxR alone is insufficient to activate the toxT promoter (Higgins and DiRita, 1994). Furthermore, recent evidence from $V$. cholerae showed TcpP to be required for the expression of toxT (Carroll et al., 1997; Häse and Mekalanos, 1998). Thus, we sought to determine whether TcpP could activate toxT transcription in $E$. coli and whether ToxR played a role in toxT activation.

An E. coli strain harbouring a chromosomally located toxT-lacZ fusion (DH92; Higgins and DiRita, 1994) was transformed with an IPTG-inducible plasmid harbouring the $t c p P H$ locus of classical $V$. cholerae strain 0395 and compared with the strain carrying the vector alone (pMMB66EH; Fürste et al., 1986). TcpPH was able to activate the toxT promoter strongly, inducing activation approximately eightfold, whereas plasmid alone had no effect (Fig. 1A; pACYC + TcpPH). A similar induction was seen when the tcpPH locus from El Tor strain E7946 was used (data not shown). In order to investigate the effect of ToxR on this TcpPH-dependent activation, we introduced ToxRS into the $E$. coli reporter strain in the presence or absence of $\mathrm{TcpPH}$. As shown previously, ToxRS alone (ToxRS + pMMB66EH) repressed activation in this background twofold (Higgins and DiRita, 1994), reflecting the fact that ToxR binds to the toxT promoter but cannot activate transcription, and actually inhibits basal transcription (Fig. 1A; Higgins and DiRita, 1994). When ToxRS and TcpPH were co-expressed, activation mediated by $\mathrm{TcpPH}$ was consistently enhanced (from 1250 to 1800 units in Fig. 1A). Activation resulting from low-level TcpPH expression in the absence of IPTG in conjunction with ToxRS was also slightly elevated over that observed with uninduced TcpPH alone (Fig. 1A; ToxRS + TcpPH -IPTG). Thus, ToxR and TcpP in combination give maximal activation of the toxT promoter.

Two other ToxR-regulated promoters, ompU and $c t x A$, were examined for TcpP-dependent activation by expressing TcpPH, ToxRS or both pairs of proteins in an E. coli strain harbouring ompU-lacZ (AC174; Crawford et al., 1998) or ctxA-lacZ (VJ787; A. Bock and V. J. DiRita, unpublished) at the same chromosomal location as the

(c) 2000 Blackwell Science Ltd, Molecular Microbiology, 38, 67-84 
toxT-lacZ fusion described above (Elliott, 1992). ToxRS activated the ompU-lacZ fusion to high levels ( $\approx 100$-fold) as seen previously (Crawford et al., 1998), whereas $\mathrm{TcpPH}$ failed to activate ompU (Fig. 1B). In addition, no enhancement of ToxR-dependent activation of $o m p U$ was seen in the presence of TcpPH (Fig. 1B). Thus, TcpPH appears to play no role in ompU regulation, which could be predicted from the observation that ompU is in the ToxT-independent branch of the $V$. cholerae ToxR regulatory cascade (Champion et al., 1997).

As the $c t x A B$ promoter is also known to be activated by ToxR in E. coli, we investigated whether TcpP would have any effect on its activation. Strains expressing ToxRS gave strong ( $\approx 30$-fold) activation of the $c t x A B$ promoter (Fig. 1C). However, expression of $\mathrm{TcpPH}$ alone or in combination with ToxRS had no effect on transcription. Taken together, we conclude that functional interaction between ToxR and TcpP for transcriptional activation is restricted to the toxT promoter.
Expression of both TcpPH and ToxRS is required for maximal TcpA and toxin production in $\mathrm{V}$. cholerae

Previous studies have shown TcpP is required for toxT, tcp $A$ and ctxAB activation in $V$. cholerae (Carroll et al., 1997; Häse and Mekalanos, 1998; Murley et al., 1999; Yu and DiRita, 1999). In order to assess the molecular basis for this observation, we constructed a $\Delta t c p P$ mutant of $V$. cholerae for use in the experiments described below. As expected, the $\Delta$ tcp $P$ derivative was defective in both TcpA expression and toxin production (Fig. 2, lane 2), whereas OmpU production was unaffected (data not shown). Plasmid complementation with tcpPH restored both TcpA and toxin production to greater than wild-type levels, suggesting that TcpP is the limiting factor in this system (Fig. 2, lane 3).

Based on the evidence from $E$. coli shown above, TcpP appears to be sufficient for the activation of toxT, albeit not maximal activation. To determine the role of ToxR in
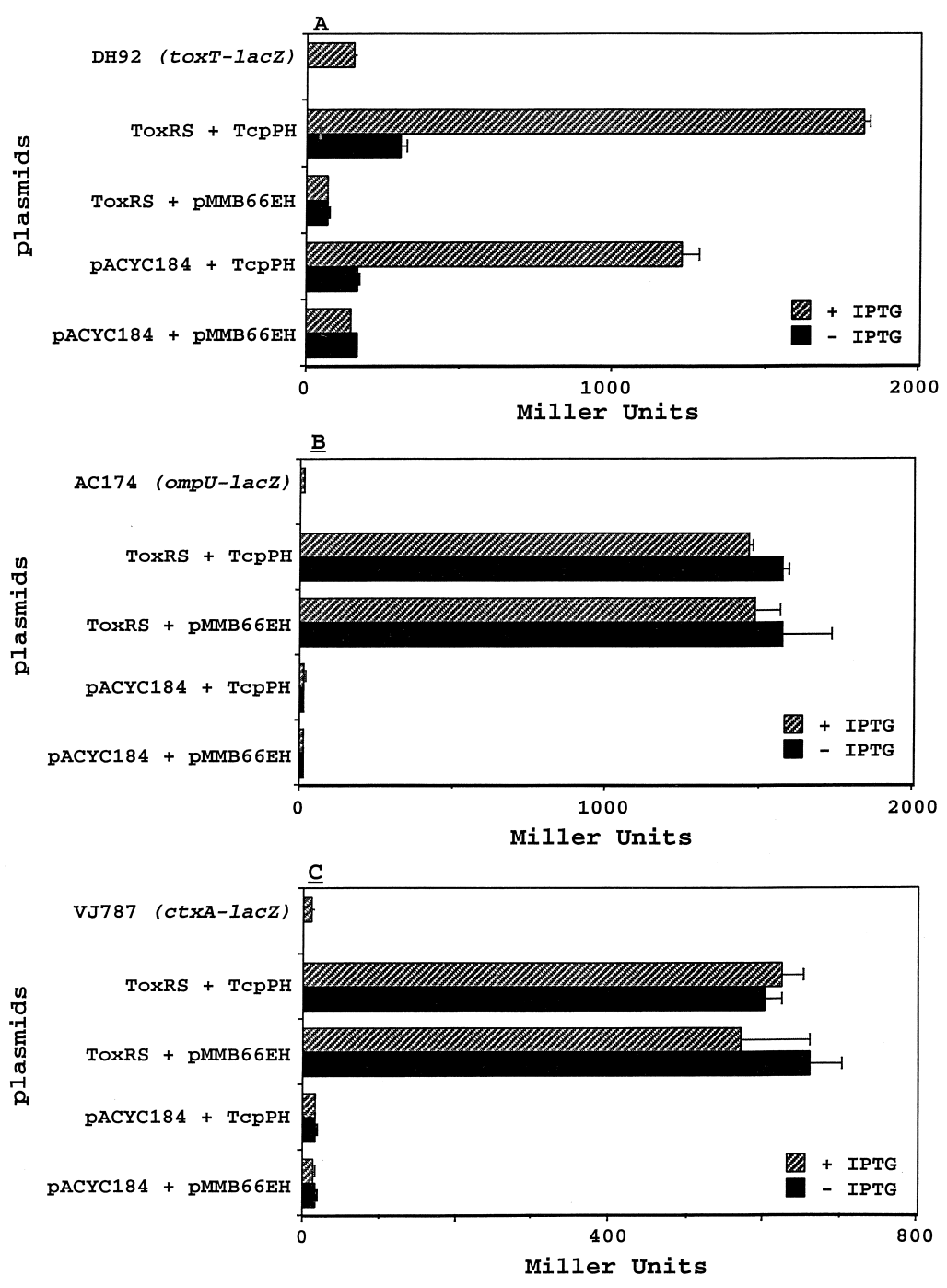

Fig. 1. TcpP-mediated activation of toxT-lac $Z$ (A), ompU-lacZ (B) and ctxAB-lacZ (C) fusions located on the $E$. coli chromosome at the put operon locus (Elliott, 1992; Higgins and DiRita, 1994; Crawford et al., 1998; A. Bock and V. J. DiRita, unpublished observations). Plasmids encoding $\mathrm{TcpPH}$ under the control of an IPTG-inducible promoter or vector alone (pMMB66EH) were co-transformed with a constitutive expression plasmid encoding ToxRS ( $p$ V21) or an empty vector (pACYC184) into the appropriate $E$. coli lacZ fusion strain. Co-expression of toxRS allowed for the analysis of TcpPH-ToxRS synergistic effects. 




Fig. 2. Effect of ToxR on virulence gene expression. $V$. cholerae mutant strains were grown overnight in LB and $1 \mathrm{mM}$ IPTG at $30^{\circ} \mathrm{C}$ in the presence of an inducible plasmid harbouring $t c p P H$ or empty vector. Cell supernatants were used to measure cholera toxin levels, and cell pellets were used for TcpA Western blotting (Experimental procedures). In order to see TcpA in the tcpPH-complemented $\Delta t c p P \Delta$ toxR strain, 10 -fold more cell extract was loaded (lanes 8 and 9).

enhancing toxT expression in its natural background, we constructed $\Delta$ tox $R$ and $\Delta$ tox $\Delta \Delta$ tcp $P$ strains of $V$. cholerae. A $\Delta$ tox $R$ mutant (EK307) was partially complemented for TcpA and toxin production by overexpressing TcpPH from a plasmid (Fig. 2; lane 5), although expression levels of both cholera toxin and TcpA were far lower than those in the presence of ToxR (Fig. 2; lanes 1 and 3 versus lane 5). A $\Delta$ toxR $\Delta$ tcpP double mutant (EK459) complemented with $\mathrm{TcpPH}$ expressed even less cholera toxin and TcpA than the single $\Delta$ tox $R$ mutant, presumably reflecting lower overall TcpP levels resulting from the lack of chromosomally encoded TcpP in conjunction with plasmidexpressed TcpP (Fig. 2, lanes 5, 7 and 8). From these data, it is clear that, although TcpP is sufficient for intermediate levels of activation of the tox $T$ promoter, ToxR acts to enhance this activation strongly.

\section{TcpP interacts on the toxT promoter downstream of ToxR}

As ToxR has been shown to interact with a region of the toxT promoter upstream of the -73 position, yet is unable to activate this promoter (Higgins and DiRita, 1994; Li et al., 2000), we next sought to gain a more mechanistic understanding of the roles of both TcpP and ToxR in toxT promoter activation. To determine the tox $T$ promoter sequences required for TcpP or ToxR interaction, a series of toxT-lacZ promoter deletion constructs was analysed in the $V$. cholerae $\Delta$ tcpP $\Delta$ tox $R$ mutant for the ability of either TcpP or ToxR expressed from a plasmid to activate transcription. Four previously characterized deletion constructs were analysed, with $5^{\prime}$ end-points at nucleotides -172 (full length), $-114,-73$ and -46 . A -172 derivative truncated to delete the -10 consensus RNA polymerase (RNAP) binding region $(\Delta-10)$ was also analysed (Fig. 3A; Higgins and DiRita, 1994). All constructs had nucleotide +45 as a $3^{\prime}$ end-point, except the $\Delta-10$ derivative.

TcpPH activated the $-172,-114$ and -73 promoter constructs when expressed from a plasmid in the
$\Delta$ toxR $\Delta$ tcp $P$ background. Neither the -46 nor $\Delta-10$ construct could be activated by TcpPH (Fig. 3B). ToxR binding to the toxT promoter was monitored as repression of basal toxT-lacZ activation, because twofold repression of the tox $T$ promoter has been documented previously in $E$. coli and is presumed to reflect ToxR binding and repression of a weak cryptic promoter upstream of the toxT promoter (Higgins and DiRita, 1994). In the absence of TcpP, overexpressed ToxRS repressed $\beta$-galactosidase activity of only the -172 and -114 promoter constructs (Fig. $3 \mathrm{C}$ ), in agreement with data mentioned above demonstrating that ToxR requires sequences upstream of -73 in the toxT promoter to activate its expression in V. cholerae (Higgins and DiRita, 1994). Thus, TcpP is able to interact functionally with a toxT promoter deleted for the ToxR binding site, suggesting that the TcpP binding site is downstream of that for ToxR.

The tox $T-l a c Z$ deletion plasmids were next introduced into the $\Delta$ tcp $P$ and $\Delta$ tox $R$ single mutant strains to assess the role of ToxR on toxT promoter activation in $V$. cholerae. The $\Delta$ tox $R$ mutant failed to activate any of the five toxT promoter constructs, demonstrating that Tox $R$ is required for toxT activation at wild-type TcpP expression levels (Fig. 3D). This is in agreement with previous studies using a different $\Delta$ tox $R$ mutant strain (JJM43; Higgins and DiRita, 1994). Overexpressed TcpP provided from a plasmid was able to restore an intermediate level of toxT activation to the $\Delta$ toxR strain (Fig. 3D).

None of the toxT-lac $Z$ deletion derivatives were activated in the $\Delta t c p P$ mutant background (Fig. 3D). Complementation of the $\Delta t c p P$ strain with plasmid-encoded tcpPH restored activation to high levels (Fig. 3D). Most notably, activation of the full-length toxT promoter $(-172)$ and the -114 deletion construct, both of which harbour the ToxR binding site, was to much higher levels in this background compared with the $\Delta$ tox $R$ and $\Delta$ tox $R \Delta$ tcp $P$ mutant strains. This enhanced activation is probably caused by the action of ToxR in the $\Delta t c p P$ background, allowing maximal activation of ToxR-recognized promoters 
A
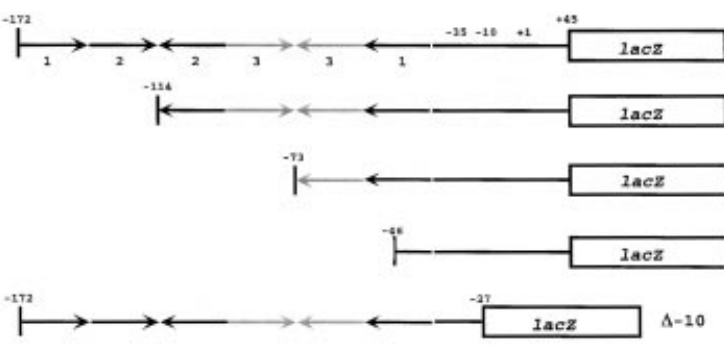

B

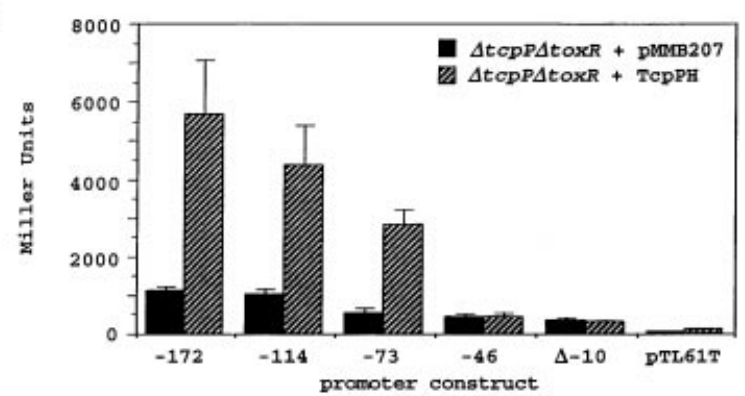

C

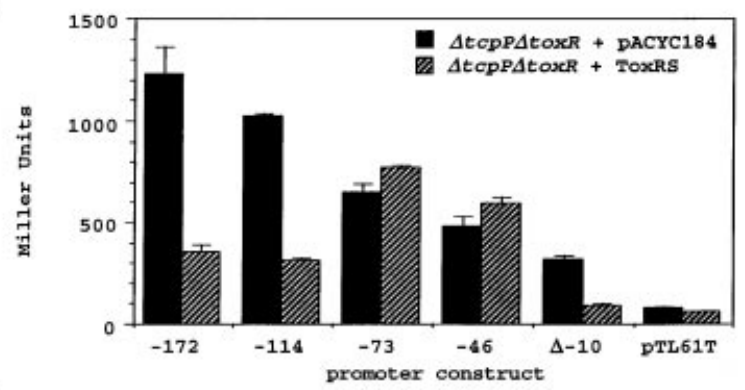

D

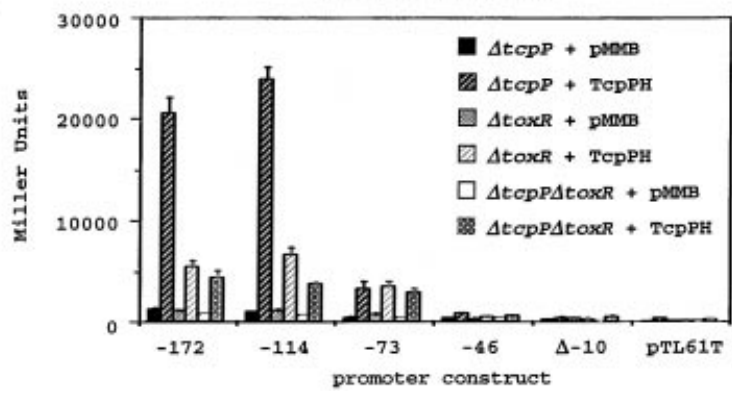

Fig. 3. Activation of various toxT-lacZ deletions.

A. Structure of toxT promoter deletion constructs (Higgins and DiRita, 1994).

B. TcpPH-directed activation of toxT-lacZ in V. cholerae. An inducible $t c p P H$-encoding plasmid or vector alone (pMMB207) in conjunction with various toxT-lacZ fusion plasmid constructs were introduced into a $V$. cholerae $\Delta$ tcpP $\Delta$ toxR strain (EK459), and $\beta$-galactosidase activity was measured after a $4 \mathrm{~h}$ induction with $1 \mathrm{mM}$ IPTG at $30^{\circ} \mathrm{C}$.

C. ToxR-dependent repression of the toxT promoter in $V$. cholerae. A constitutive toxRS-encoding plasmid or vector alone (pACYC184) in conjunction with various toxT-lacZ fusion plasmid constructs was introduced into a $V$. cholerae $\Delta$ tcpP $\Delta$ toxR strain (EK459). Cells were diluted 1:100 from an overnight culture, induced for $4 \mathrm{~h}$ at $30^{\circ} \mathrm{C}$, and $\beta$-galactosidase activity was measured.

D. The effect of ToxR on toxT-lacZ induction by TcpP. An inducible plasmid encoding tcpPH or vector alone (pMMB207) in conjunction with various toxT-lacZ fusion plasmid constructs was introduced into $V$. cholerae $\Delta$ tcpP (RY1), $\Delta$ toxR (EK307) and $\Delta$ tcpP $\Delta$ toxR (EK459) strains. Cells were induced with $1 \mathrm{mM} \mathrm{IPTG}$ for $4 \mathrm{~h}$ at $30^{\circ} \mathrm{C}$, and $\beta$-galactosidase activity was measured.
$(-172$ and -114$)$ by TcpP, and is similar to tox $T-l a c Z$ expression levels seen in the wild-type strain 0395 (data not shown; Higgins and DiRita, 1994).

Together, these data demonstrate that ToxR and TcpP interact with the toxT promoter at distinct positions required for activation. ToxR is positioned upstream of -73 , whereas TcpP requires a site downstream of -73 . This would place TcpP in a position closer to the basal promoter elements and presumably in closer proximity to RNAP than ToxR. Our data also suggest that TcpP is the direct activator in this system and that ToxR provides a supporting role, as evidenced by the facts that TcpP overexpression alleviates Tox $R$ dependence and ToxR alone cannot active toxT expression.

\section{TcpP-and ToxR-dependent toxT promoter interactions}

To assess the DNA-binding properties of TcpP and ToxR on the tox $T$ promoter, membranes from four $V$. cholerae derivatives were prepared and analysed by an electrophoretic mobility shift assay (EMSA) using the -172 to +45 tox $T$ fragment as a probe. The strains used were O395 (wild type), EK307 ( $\Delta$ toxR), RY1 ( $\Delta$ tcpP) and EK459 $(\Delta$ tcp $\Delta \Delta$ tox $R$ ). Membranes were prepared from cells grown under conditions that induce high levels of toxin and TCP pilus expression (Miller et al., 1987; Miller and Mekalanos, 1988). Whereas membranes lacking both TcpP and ToxR showed negligible binding to the -172 to +45 tox $T$ promoter construct, even at the highest concentration of total protein tested (Fig. 4; lane 4), membranes harbouring TcpP showed binding to the toxT promoter at this concentration relative to the $\operatorname{ToxR}^{-} \mathrm{TcpP}^{-}$control (Fig. 4; lane 8 versus lane 4). In contrast, membranes containing either ToxR alone or ToxR and TcpP bound the tox $T$ probe at 10 -fold lower concentrations of membrane protein than those with TcpP alone (Fig. 4; lanes 10 and 14), indicating that ToxR binds the toxT promoter more efficiently than TcpP under conditions in which virulence gene expression is induced. This could reflect a difference in relative DNA-binding affinities, protein expression levels or both.

\section{TcpP binds downstream of ToxR on the toxT promoter}

The tox $T-l a c Z$ expression experiments described above indicate that $\mathrm{TcpP}$ can interact functionally with the toxT promoter downstream of ToxR. We sought to test this conclusion biochemically by assessing the ability of TcpP and ToxR to bind different regions of the toxT promoter. To determine which region of the toxT promoter TcpP binds, $V$. cholerae membranes harbouring TcpP, ToxR or neither protein were analysed at $2.5 \mathrm{mg} \mathrm{ml}^{-1}$ or $10 \mathrm{mg} \mathrm{ml}^{-1}$ membrane protein for their ability to shift 


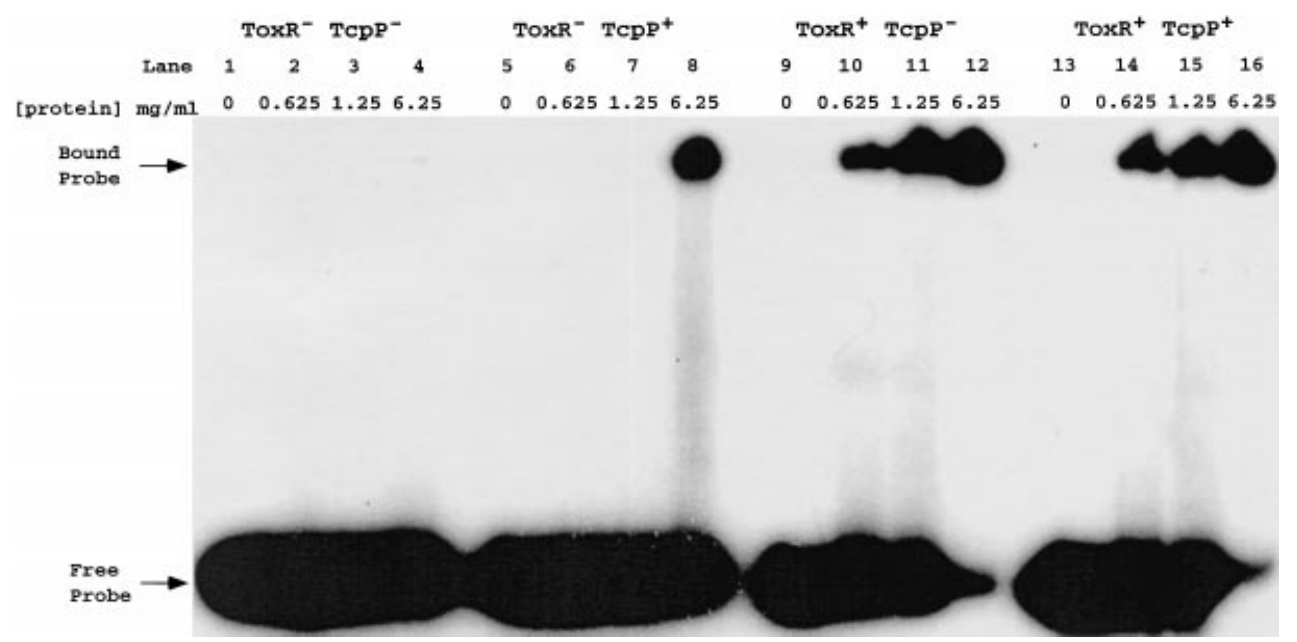

Fig. 4. Binding of full-length toxT promoter to $V$. cholerae cellular membranes containing ToxR, TcpP or neither protein. End-labelled probe containing the -172 to +45 region of the tox $T$ promoter was incubated with increasing concentrations of membrane for 30 min at $30^{\circ} \mathrm{C}$ and resolved in a $6 \%$ non-denaturing polyacrylamide gel. The ToxR and TcpP proteins were expressed from genes at their normal chromosomal locus.

radioactively labelled DNA fragments corresponding to the various toxT promoter deletions described above.

Membranes from a strain deleted for both tcp $P$ and tox $R$ showed low levels of binding to all probes tested, although background binding to the -172 probe at the higher concentration of membrane was significant (Fig. 5; lanes 3, 12 and 21). This increase in background binding compared with the previous experiment (Fig. 4) may reflect the fact that slightly higher concentrations of membrane were used $\left(10 \mathrm{mg} \mathrm{ml}^{-1}\right.$ versus $6.25 \mathrm{mg} \mathrm{ml}^{-1}$ ) or the inherent variability in the assay from experiment to experiment. Membranes containing TcpP, but not ToxR, bound each promoter construct more readily than the $\mathrm{TcpP}^{-} \mathrm{ToxR}^{-}$control, except that in which the -46 to -73 region was deleted (Fig. 5; lanes 5, 14 and 23; -46 to +45 data not shown). Consistent with the binding experiment using a full-length toxT promoter (Fig. 4), the
$\mathrm{TcpP}^{+} \mathrm{ToxR}^{-}$membranes showed weak promoter interaction at $2.5 \mathrm{mg} \mathrm{ml}^{-1}$ membrane protein, but bound well at $10 \mathrm{mg} \mathrm{ml}^{-1}$ protein. The membrane preparation from a strain expressing only ToxR, on the other hand, failed to shift promoters lacking the -73 to -114 region (Fig. 5 , lane 25), but did shift the full-length and -114 deletion tox $T$ fragments even at low membrane concentrations (Fig. 5, lanes 6 and 15). Membranes from the wild-type strain $\mathrm{O} 395$ also failed to interact above background with the -73 deletion fragment, even at the higher concentration, despite the fact that TcpP protein was presumably present. One possible explanation is that TcpP protein in the cell may be complexed with ToxR and, as this promoter lacks the ToxR binding site, TcpP could be sequestered away from the DNA. In the end, the biochemical evidence confirms the genetic activation data in establishing the TcpP binding site as $3^{\prime}$ to the

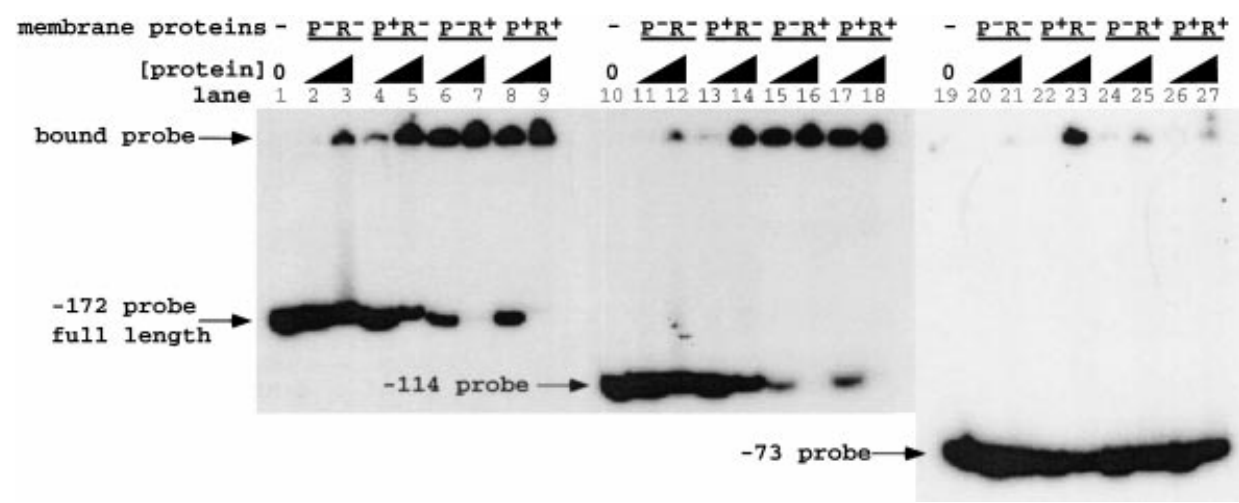

Fig. 5. toxT promoter deletion gel mobility shift assays. Increasing amounts of cell membrane derived from $V$. cholerae strains was mixed with end-labelled toxT probes for $30 \mathrm{~min}$ at $30^{\circ} \mathrm{C}$ and subjected to non-denaturing PAGE. Protein-probe complexes were retained in the well, whereas promoter deletion constructs ran into the gel according to size. Protein concentrations used were $0,2.5$ or $10 \mathrm{mg} \mathrm{ml}^{-1} \mathrm{membrane}$ protein. The presence or absence of either ToxR $(R)$ or TcpP $(P)$ in each membrane preparation is denoted with either '+' or '-' respectively. 
A

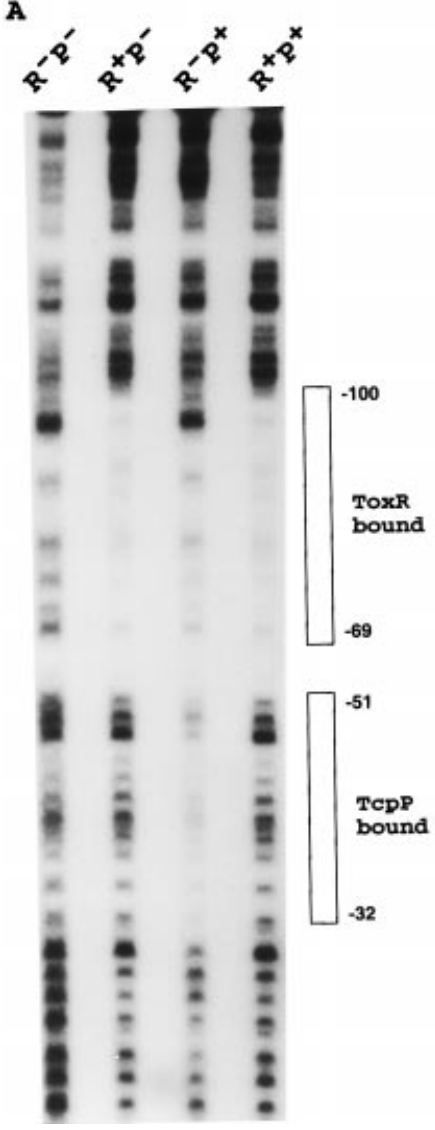

B

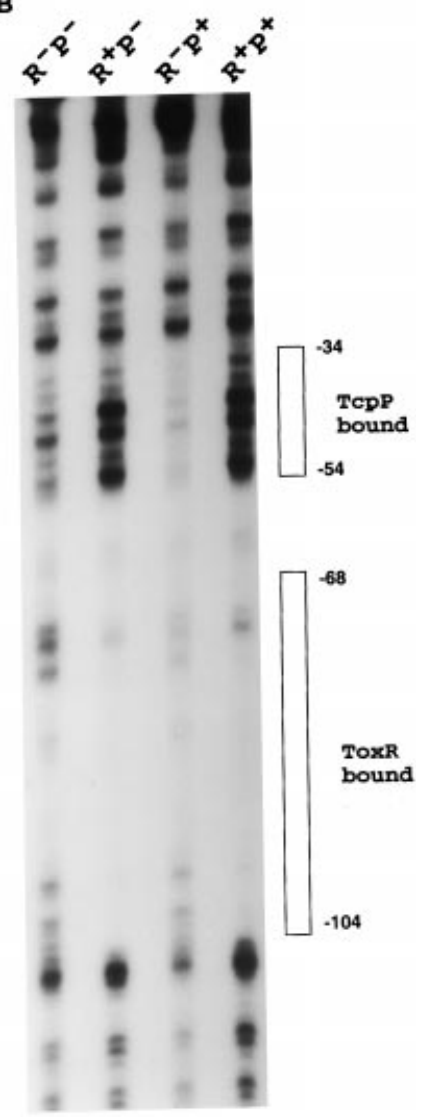

Fig. 6. DNase I footprint analysis of the toxT promoter. $V$. cholerae membrane extract (20 $\mathrm{mg} \mathrm{ml}^{-1}$ ) derived from strains expressing ToxR and/or TcpP or neither protein were mixed with the -172 to +45 toxT promoter construct in which the top strand $(A)$ or bottom strand $(B)$ had been end labelled. After digestion with DNase I, samples were subjected to electrophoresis in a $6 \%$ polyacrylamide denaturing gel (Experimental procedures).

ToxR binding site and places TcpP in close proximity to the putative RNAP consensus binding site.

\section{TcpP-containing membranes protect a region immediately upstream of the -35 consensus site of the toxT promoter from DNase I digestion}

In order to characterize the precise TcpP binding site within the toxT promoter further, membranes prepared for

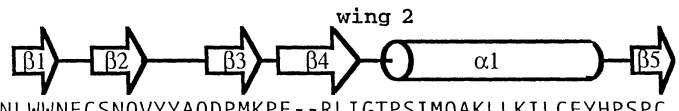

MGYVRVIYOFPDNLWWNECSNQVYYAODPMKPE--RLIGTPSIMQAKLLKILCEYHPSPC MSHI GTKF I LAEKFTFDPLSNTLIDKEDSEEI IRL GSNESRI LWLLAQRPNEVI MSHCVVLNKLESVLI I GD - SRY - -ALSKNEVLLLECLYLRAGDVI MOQPVVRVGEWLVTPSINOISRNGROLTLEPRLIDLLVFFAQHSGEVL MPHFNPVPVSNKKFVFDDFI - - LNMDGSLVRSE - -KKVNIPPK-EYAVLVILLEAAGEIV MRRISPMAVEEVIEMQGLSLDPTSHRVMAGEEP - - LEMGPT - -EFKLLHFFMTHPERVY

PGAPSQEEAVIAFGKFKLNLGTREMFREDEP-- -MPLTSG- -EFAVLKALVSHPREPL the EMSAs discussed above were used to generate toxT promoter footprints. Membranes containing TcpP, but lacking ToxR, protected a region of the top strand of the tox $T$ promoter extending from nucleotides -51 to -32 , including the $3^{\prime}$ half of the previously identified inverted repeat 1 within the toxT promoter (Fig. 6A, lane 3; Fig. 3A; Higgins et al., 1992). Somewhat weaker protection by TcpP-containing membranes extended even further upstream to about nucleotide -80 . Footprinting
TcpP (V.c.) ToxR (V.C.) PsaE (Y.p.) CadC (E.C.) HilA (S.t.) PhoB (E.C.) OmpR (E.C.)
Fig. 7. Linear protein alignments of TcpP, ToxR and related proteins. Only the $\mathrm{N}$-termini (TcpP, ToxR, PsaE and CadC) or C-termini (HilA, PhoB and OmpR) are shown. The secondary structure domains of $\mathrm{OmpR}$, based on the crystal structure of OmpR (MartínezHackert and Stock, 1997a), are indicated above the sequence. TcpP residues targeted for mutagenesis are indicated by *. Proteins were aligned according to PILEUP analysis.

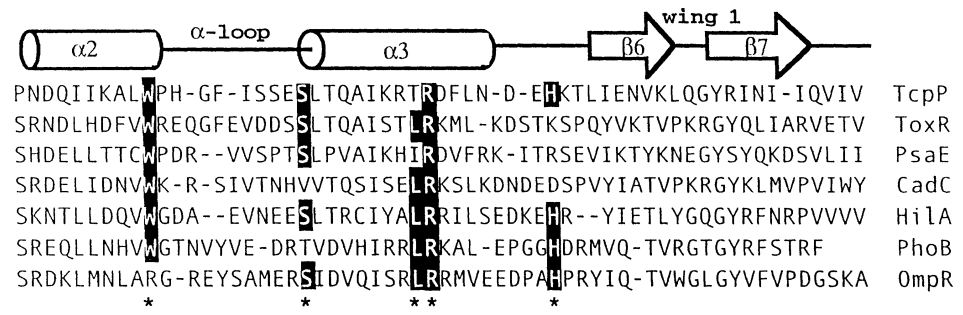

(c) 2000 Blackwell Science Ltd, Molecular Microbiology, 38, 67-84 
A
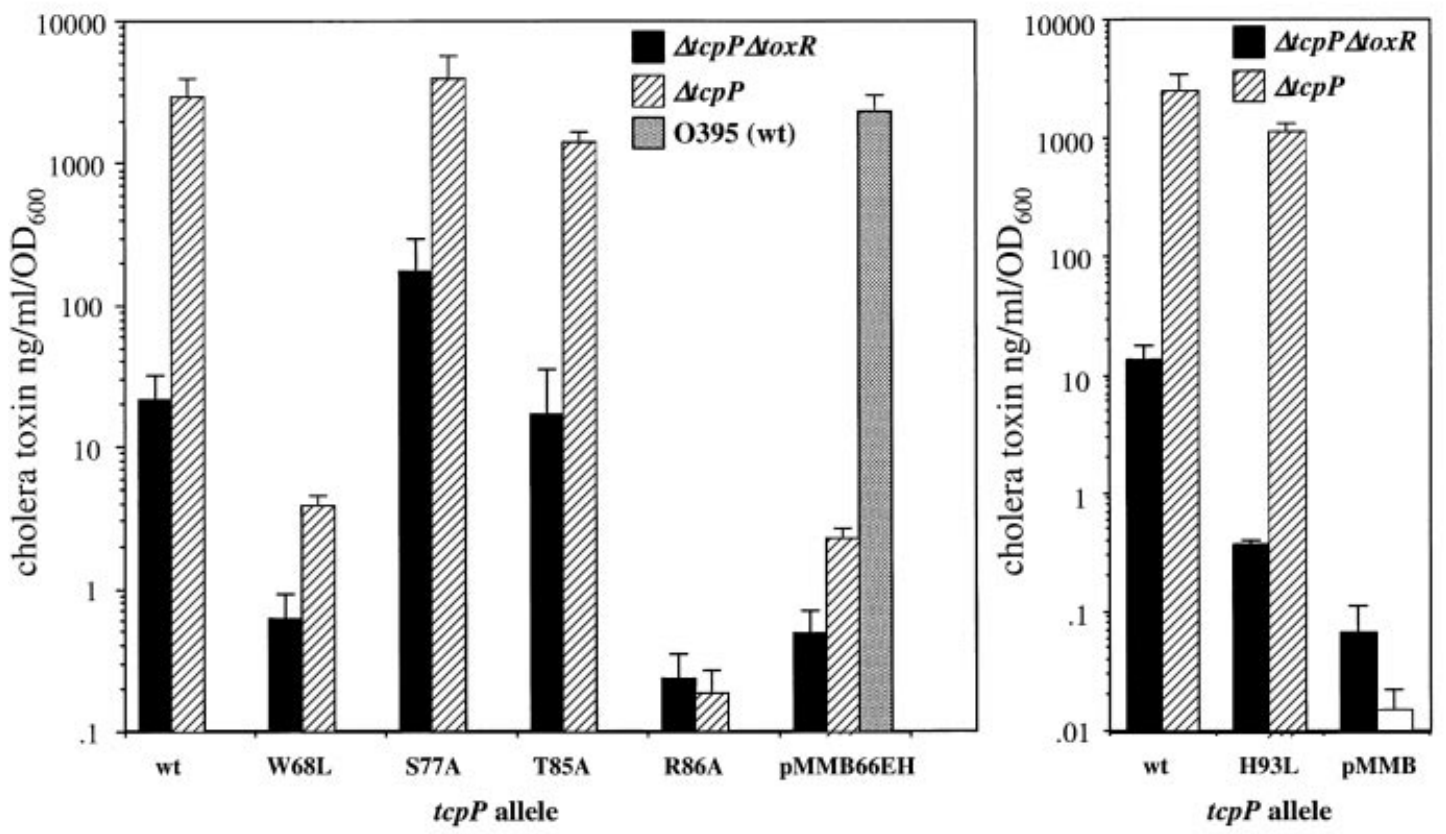

B

$\Delta t c p P / \Delta t o x R$

$\Delta t c p P$

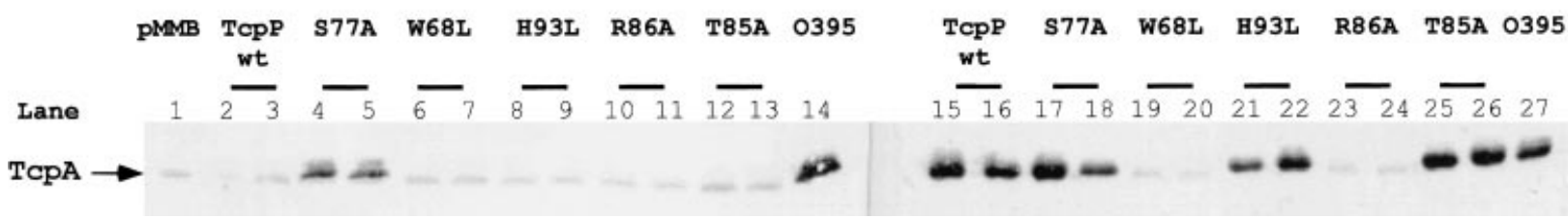

Fig. 8. Expression of cholera toxin (A), TcpA (B) or tox $T(C)$ after complementation of a $\Delta t c p P$ strain with various tcpP alleles in the presence or absence of ToxR, and DNA-binding activities of TcpP mutant proteins (D).

A. V. cholerae cells expressing tcpP from an inducible plasmid (pMMB66EH) were grown overnight at $30^{\circ} \mathrm{C}$ in LB containing $1 \mathrm{mM} I P T G$, and supernatants were analysed for toxin production in a GM - ELISA assay, as described in Experimental procedures.

B. Cells from overnight IPTG-induced cultures were resuspended in $1 \times$ Laemmli buffer, run on a 15\% SDS-polyacrylamide gel, and TcpA was detected with an anti-TcpA antibody by Western blot analysis (Experimental procedures).

C. Plasmid-encoded (pMMB207), inducible tcpP alleles harbouring a C-terminal epitope tag were induced for $4 \mathrm{~h}$ in $V$. cholerae $\Delta t c p P$ or $\Delta t c p P \Delta$ toxR in the presence of a plasmid carrying a tox $T-l a c Z$ fusion reporter ( -172 to +45 promoter). Western blot analysis of whole-cell extracts are reported below $\beta$-galactosidase activities using an anti-HSV antibody to detect the levels of each epitope-tagged derivative in the absence or presence of ToxR.

D. Membranes derived from EK459 ( $\Delta$ tcpP $\Delta$ toxR) expressing wild-type HSV-tagged TcpP or various mutants were bound to toxT promoter fragments using either $4 \mathrm{mg} \mathrm{ml}^{-1}$ or $10 \mathrm{mg} \mathrm{ml}^{-1}$ total membrane proteins as described in Experimental procedures. The -73 to +45 construct contains the TcpP binding site, whereas the -46 to +45 does not. Western blot analysis of the relative levels of each TcpP mutant used for gel shift analysis is shown under the appropriate lane. In the cases of TcpP-wt, TcpP-W68L, TcpP-S77A and TcpP-T85A, 2-mg ml ${ }^{-1}$ and $5 \mathrm{mg} \mathrm{ml}^{-1} \mathrm{TcpP}$ containing membranes were doped with $2 \mathrm{mg} \mathrm{ml}^{-1}$ and $5 \mathrm{mg} \mathrm{ml}^{-1}$ negative control membranes (pMMB207) to achieve identical membrane concentrations and comparable TcpP levels. For pMMB207, TcpP-R86A and TcpP-H93L lanes, $4 \mathrm{mg} \mathrm{ml}^{-1}$ and $10 \mathrm{mg} \mathrm{ml}^{-1}$ undoped membranes were used (Experimental procedures).

with membranes containing either ToxR alone or ToxR and TcpP gave a toxT protection pattern indistinguishable from that observed previously with $E$. coli membranes containing ToxRS, protecting a region from -100 to -69 and characterized most strongly by protection of a hypersensitive site around position -92 (Fig. 6A, lanes 2 and 4; Li et al., 2000). Similar regions within the bottom strand of the toxT promoter were also protected, although the boundaries were slightly different (Fig. 6B). On the bottom strand, ToxR binding leads to protection of two hypersensitive sites centring around position -75 . ToxR also appears to lead to enhanced cleavage within the TcpP binding site, suggesting that ToxR binding may lead to an alteration in promoter architecture (Fig. 6B). These data confirm the gel shift and promoter fusion studies, in which TcpP interacts with a region of the tox $T$ promoter 
C
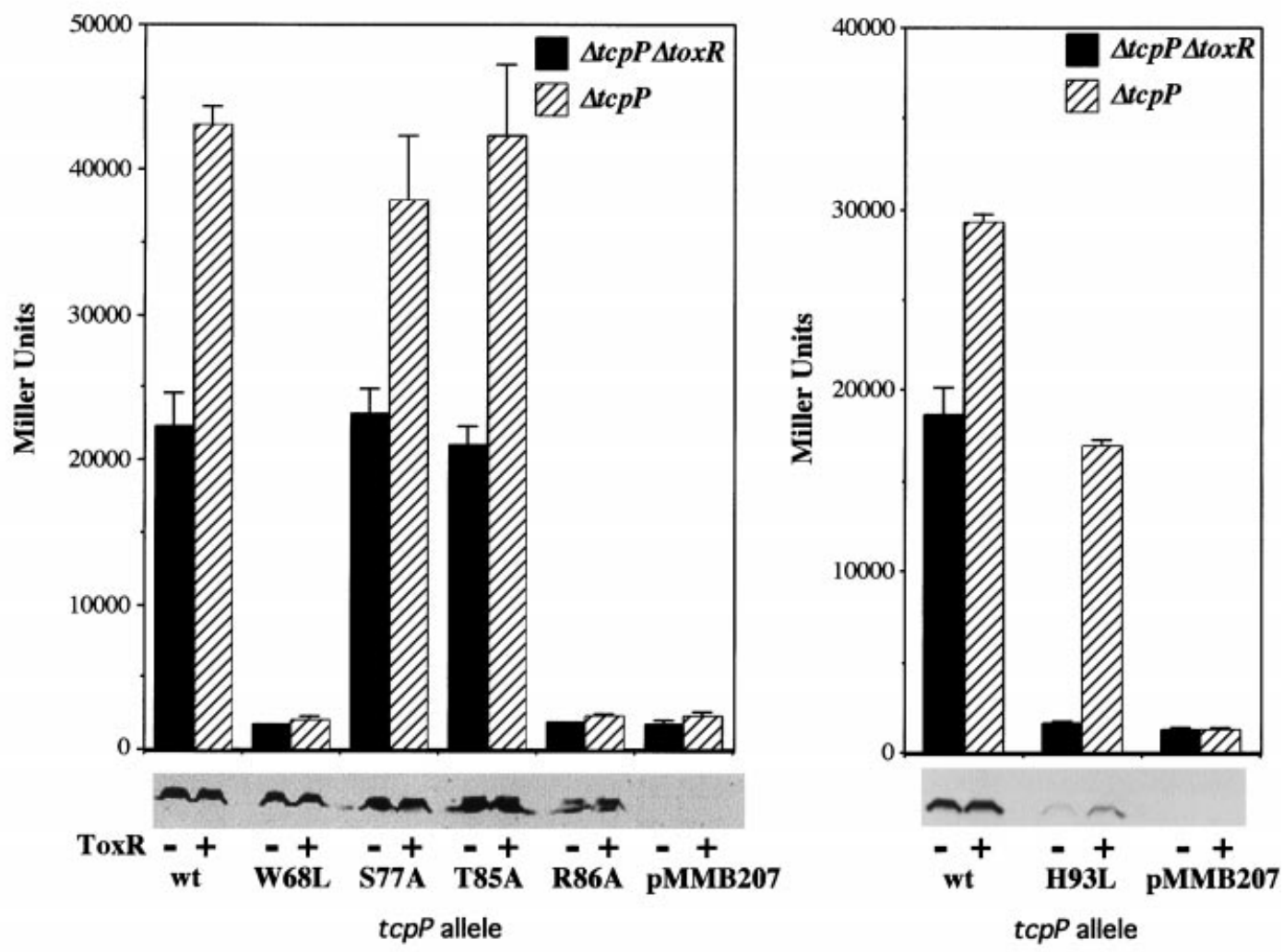

D

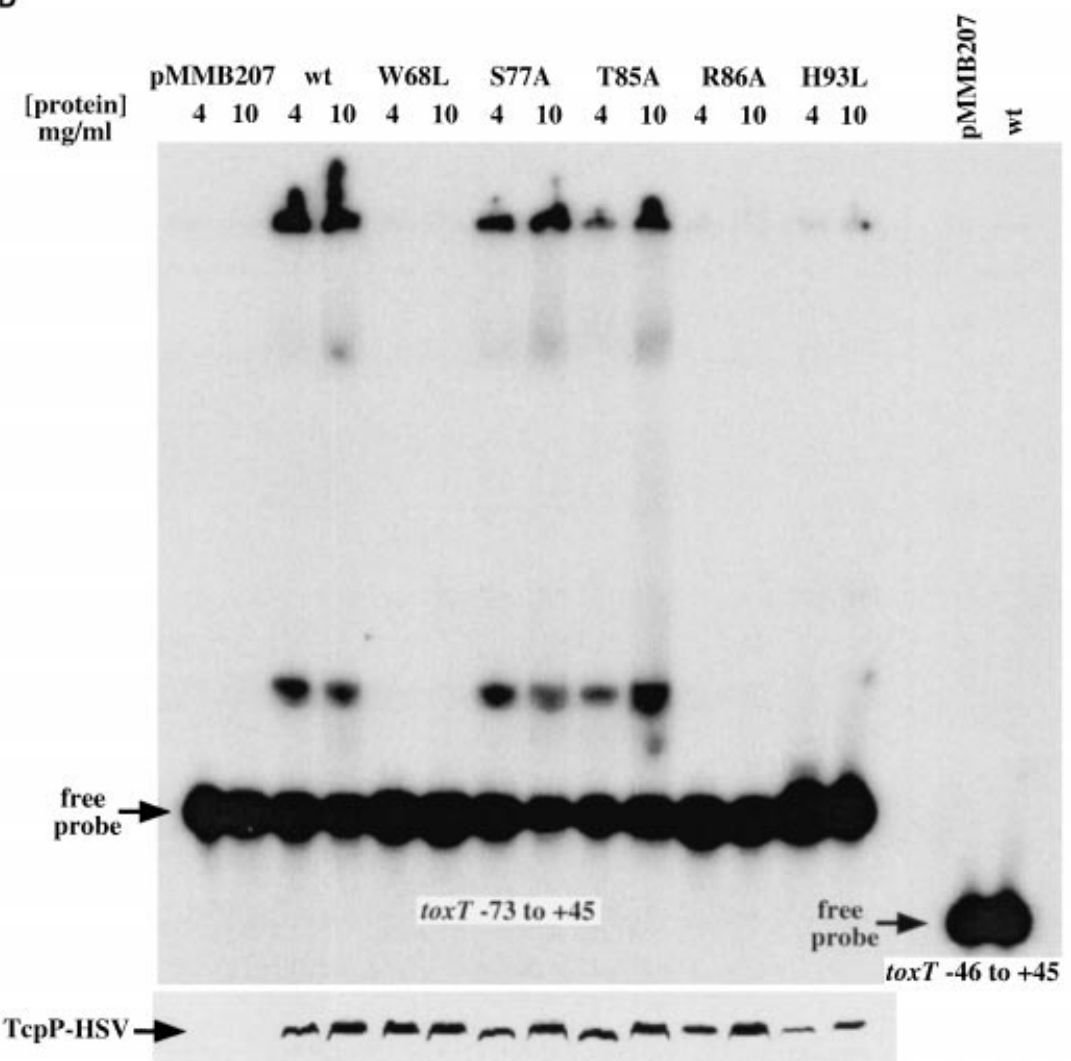

Fig. 8. Continued.

(c) 2000 Blackwell Science Ltd, Molecular Microbiology, 38, 67-84 
just $5^{\prime}$ to the RNAP consensus binding site, with ToxR binding to a region just upstream. The inability of TcpP to protect the probe in the presence of ToxR was unexpected (Fig. 6A, lane 4) and is addressed below.

\section{ToxR expression suppresses the activation defect of a TcPP DNA-binding mutant}

One possibility to account for the fact that $V$. cholerae membranes containing both TcpP and ToxR did not protect the region from -51 to -32 characteristic of TcpP alone (Fig. 6A, lane 4) is that, in the presence of ToxR, TcpP may bind DNA poorly, even though it is capable of activating transcription. This reduction in binding may result from alteration of the TcpP binding site architecture after ToxR binding upstream or ToxR-TcpP protein interactions that result in a TcpP conformation less able to bind DNA. To test this hypothesis, we generated a number of tcpP site-directed mutants based on mutant phenotypes of homologous OmpR-like DNA-binding proteins (Fig. 7) with the goal of obtaining derivatives defective for DNA binding or RNAP interaction.

Five TcpP derivatives were constructed by mutating a region of the protein predicted to be important for DNA binding or RNAP interaction (Ottemann et al., 1992; Russo et al., 1993; Pratt and Silhavy, 1994; Makino et al., 1996; Martínez-Hackert and Stock, 1997a; Fig. 7). These derivatives were expressed in $V$. cholerae in either the presence or the absence of endogenous levels of ToxR to assess their dependence on ToxR for transcriptional activation. If the above hypothesis is correct, a DNAbinding mutant of TcpP might retain its ability to activate transcription provided that ToxR is present. As an indirect measure of tox $T$ expression, toxin and TcpA production resulting from toxT activation was assessed.

Two mutants, TcpP-W68L and TcpP-R86A, were defective for toxin and TcpA production regardless of the ToxR status of the cell (Fig. $8 \mathrm{~A}$ and $\mathrm{B}$ ). Although by analogy with OmpR, TcpP-W68 (Trp-68) resides at the edge of the $\alpha$-loop of TcpP, hypothesized to be involved in direct RNAP interaction (Martínez-Hackert and Stock, 1997b), this protein was unable to bind the toxT promoter (Fig. 8D). Arginine-86 of TcpP was predicted to be involved in DNA binding, as some mutations in the homologous residue of ToxR disrupt promoter binding, although these defects depended on the nature of the amino acid substitution (Ottemann et al., 1992). Consistent with its defect in toxin and TcpA production, TcpPR86A failed to bind toxT promoter DNA (Fig. 8D).

Two other mutants, TcpP-S77A and TcpP-T85A, when expressed from a plasmid, complemented both the $\Delta t c p P \Delta$ tox $R$ and $\Delta t c p P$ strains to the same extent as wild-type TcpP (Fig. 8A and B), showing that they are not defective for either ToxR-independent or ToxR-dependent activation of toxT transcription. In addition, both proteins maintained strong DNA-binding activity, as measured using epitope-tagged versions of each TcpP derivative (Fig. 8D, Experimental procedures). Consistent with the results presented above (Fig. 2), when TcpP was overexpressed, the addition of ToxR to the system enhanced both toxin expression (50- to 100-fold; Fig. 8A) and TcpA expression (Fig. 8B). TcpP-S77A appears to direct higher levels of toxin and TcpA production than wild-type TcpP in the absence of ToxR, suggesting that it may be better able to interact with the toxT promoter than wild-type TcpP (Fig. 8A, ToxR-independent toxin production; Fig. 8B, lanes 4 and 5).

The final derivative, TcpP-H93L, stood apart in failing to direct toxin or TcpA production in the absence of ToxR, whereas in the presence of ToxR, virulence gene expression was restored to nearly wild-type levels (Fig. 8A and B). Despite the ability of TcpP-H93L to activate toxT in the presence of ToxR, TcpP-H93L was almost completely defective in DNA binding (Fig. 8D). The phenotype of TcpP-H93L shows that toxT activation may occur if DNA binding is severely attenuated, provided that ToxR is present. This suggests that an important role for ToxR in TcpP function is to provide promoter recognition for the activation domain of TcpP to stimulate toxT transcription.

To measure transcriptional activation by these various TcpP derivatives directly, plasmid-expressed epitopetagged versions of each $t c p P$ allele were introduced into $V$. cholerae $\Delta t c p P$ or $\Delta t c p P \Delta$ tox $R$ backgrounds in the presence of a second plasmid harbouring a toxT-lacZ reporter construct (toxT promoter -172 to +45 ). $\beta$ Galactosidase activities directed by the various TcpP derivatives were consistent with the results seen for toxin and TcpA production, except that toxT-lacZ expression directed by TcpP-H93L in the presence of ToxR did not reach wild-type levels (Fig. $8 \mathrm{C}$ ). Western analysis using antibody directed against the C-terminal epitope tag of each derivative showed TcpP-W68L, TcpP-S77A and TcpP-T85A to be as stable as wild-type TcpP (Fig. 8C). TcpP-R86A and TcpP-H93L were both less stable than wild-type TcpP, although it is clear that the level of TcpPH93L expressed is sufficient to activate toxT expression strongly in the presence of ToxR (Fig. 8C). The presence of ToxR also appears to stabilize TcpP-H93L protein partially (Fig. 8C), an observation that will be discussed later.

\section{A ToxR substitution, ToxR-G80S, results in reduced OmpU production, but wild-type toxin and TcpA levels}

ToxR is believed to be the direct activator of ompU in $V$. cholerae (Crawford et al., 1998), whereas the results above suggest that TcpP is the direct activator of toxT. To determine whether the ability of ToxR to activate

(c) 2000 Blackwell Science Ltd, Molecular Microbiology, 38, 67-84 
A

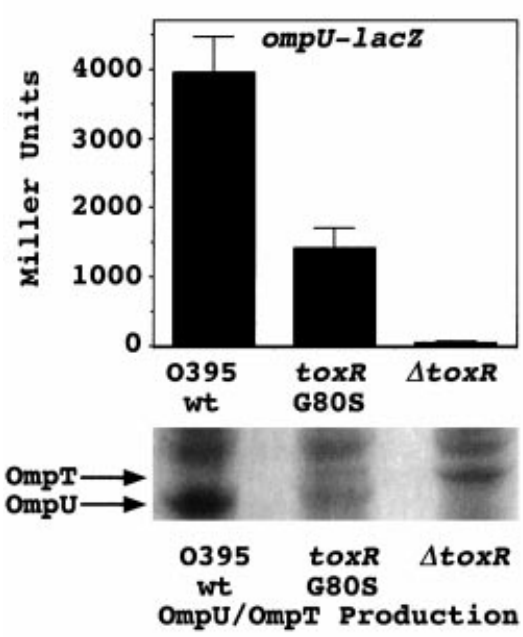

C

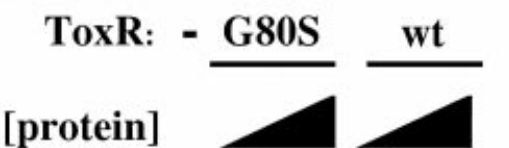

[protein]

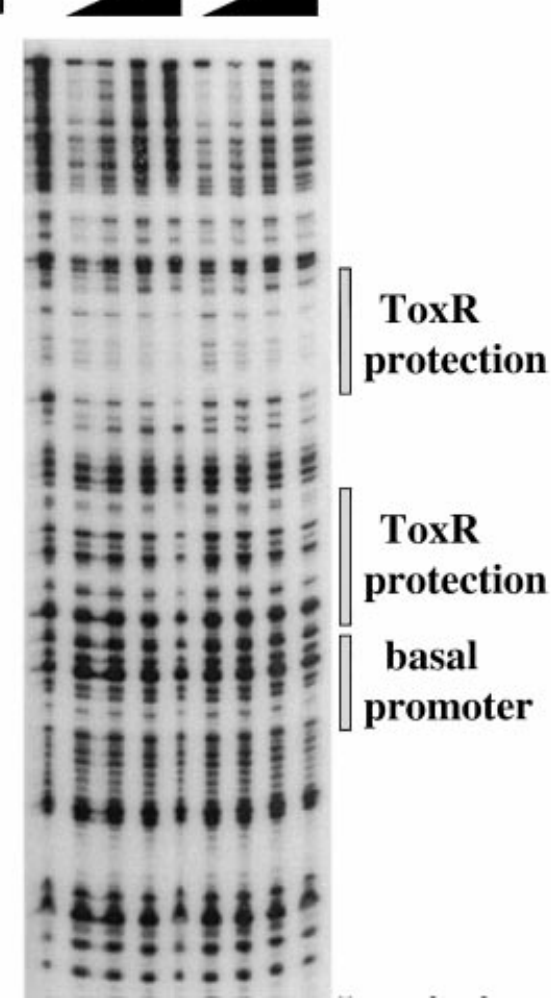

transcription is required at the tox $T$ promoter, a tox $R$ substitution mutant was constructed, toxR-G80S, based on the activation defect of a similar mutation characterized in the related protein, OmpR (Russo et al., 1993). Replacement of the wild-type toxR allele with the toxRG80S allele (EK739) in a background harbouring an ompU-lacZ fusion on the chromosome led to a marked
Fig. 9. Effects of a toxR-G80S mutation on ompU and toxT expression separates differing roles for ToxR.

A. ompU-lacZ expression was measured in the presence of wild-type ToxR, ToxR-G80S, or in the absence of ToxR from a chromosomal ompU-lac $Z$ fusion. The corresponding levels of OmpU and OmpT from isogenic strains lacking the ompU-lac $Z$ reporter are shown below the activation data.

B. toxT-lacZ was measured in the presence of wild-type ToxR, ToxR-G80S, or in the absence of ToxR. toxT-lacZ expression was from a plasmid construct (pTLI2), and the vector lacking the toxT promoter (pTL61T) served as the negative control. The corresponding levels of cholera toxin production, in the absence of the tox $T-l a c Z$ fusion plasmid, are reported below the activation data.

C. ompU promoter binding by wild-type ToxR and ToxR-G80S. Footprinting analysis with increasing concentrations of membrane from strains harbouring a wild-type tox $R$ locus, toxR-G80S or $\Delta$ tox $R$ was carried out on the ompU promoter. The concentrations of membrane used were $0,1.25,2.5$ and $5 \mathrm{mg} \mathrm{ml}^{-1}$. For the $\Delta$ tox $R$ strain, only $5 \mathrm{mg} \mathrm{ml}^{-1}$ was used. reduction in the ability of ToxR to activate the ompU promoter (Fig. 9A), whereas ToxR expression levels (not shown) and DNA binding were similar to wild-type levels (Fig. 9C). Decreased activation of the ompU promoter was also reflected in the reduced levels of OmpU in the cells and an increase in OmpT levels (Fig. 9A). ompUlacZ expression was not completely abolished in the 
presence of the toxR-G80S allele, as a $\Delta$ tox $R$ derivative was decreased even further for ompU activation and OmpU protein levels (Fig. 9A). In contrast to the reduction in ompU activation, the ToxR-G80S derivative directed wild-type levels of toxT-lacZ expression from a plasmid harbouring the full-length toxT promoter fused to lac $Z$ (Fig. 9B). The effect on ompU transcription, but not that of tox $T$, was reflected in the fact that OmpU levels were decreased, whereas toxin production by ToxR-G80S was indistinguishable from wild type (Fig. 9A and B). These results suggest that the role of ToxR at the ompU promoter differs from that at toxT. Mutations that affect the transcriptional activation domain of ToxR affect ompU transcription, but have no effect on toxT expression. Thus, rather than serving as a second activator (in direct contact with RNAP) on the toxT promoter, ToxR appears to use another mechanism to enhance the ability of TcpP to activate transcription.

\section{Discussion}

The current study investigated the role of both TcpP and ToxR in toxT promoter activation. Previous work showed that ToxR is necessary, but not sufficient, for the activation of toxT (Higgins and DiRita, 1994). Experiments in $E$. coli reported here confirmed the recent observation that the expression of TcpPH is sufficient to activate a toxT-lac $Z$ fusion construct, and co-expression with ToxRS enhances this activation (Fig. 1A; Murley et al., 1999). Enhancement of TcpP-mediated toxT activation by ToxR has also been shown in $V$. cholerae (Fig. 3D; Häse and Mekalanos, 1998). This co-dependence of maximal toxT expression on TcpP and ToxR may indicate a direct interaction between these proteins, although attempts to demonstrate such an interaction have failed (C. Häse, personal communication; E. S. Krukonis, unpublished results). From these results, we conclude that TcpP is the factor previously postulated as being required for tox $T$ promoter activation (Higgins and DiRita, 1994).

Although ToxR is required for TcpP-dependent activation of the tox $T$ promoter in $V$. cholerae under wild-type conditions of tcpP expression, overexpression of tcpPH releases tox $T$ induction from complete ToxR control. This was demonstrated most clearly by the fact that the expression of $t c p P H$ alone in a $\Delta t c p P \Delta$ tox $R$. cholerae derivative (EK459) could activate a toxT-lacZ fusion construct (Fig. $3 \mathrm{~A}$ ). In addition, both $\Delta$ toxR and $\Delta$ tcpP $\Delta$ toxR mutant strains could be partially complemented for TcpA and toxin production by $t c p P H$ overexpression (Fig. 2). Thus, ToxR may enhance the interaction of TcpP with the tox $T$ promoter, an enhancement that becomes superfluous when TcpP is present at high enough concentrations, although it should be emphasized that, even under conditions of TcpP overexpression, the presence of ToxR still results in even higher levels of toxT expression (Figs 2 and 3D).

Our results also provide an explanation for previous observations regarding the position of the Tox $R$ binding site on the toxT promoter, which is considerably upstream of the basal promoter elements (Higgins and DiRita, 1994; $\mathrm{Li}$ et al., 2000; Fig. 3C, this work). Using promoter deletion constructs fused to $l a c Z$, we were able to assign the relative binding positions of TcpP and ToxR on the toxT promoter (Fig. 3B and C) by taking advantage of the fact that overexpression of TcpPH obviates the requirement of ToxR for activation. Both promoter activation experiments (Fig. 3B) and gel shift analysis using $V$. cholerae membranes (Fig. 5) demonstrated that TcpP interacts with a region of the tox $T$ promoter in close proximity to the predicted RNAP consensus binding site. Footprinting analysis in the presence of TcpP or ToxR confirmed these results and allowed us to assign the interaction sites as -51 to -32 for TcpP and -100 to -69 for ToxR. This ToxR binding site is identical to that found using membranes from E. coli expressing ToxRS as the source of ToxR ( $\mathrm{Li}$ et al., 2000). The region protected from DNase I digestion by TcpP may extend further upstream to about nucleotide -80 (Fig. 6), but such protection is weaker. This may explain why a tox $T$ promoter truncated at -73 can be activated by TcpP, yet longer constructs are even more strongly activated (Fig. 3). That the TcpP and ToxR binding sites appear to overlap in the region from nucleotides -69 to -80 of the toxT promoter may reflect the fact that both proteins contain similar OmpR-like DNA-binding domains. As the promoter binding position of TcpP is similar to that of several other transcriptional activators (Collado-Vides et al., 1991) and given the similarity between TcpP and OmpR-like proteins, we propose that TcpP activates transcription by contacting RNAP directly (Ishihama, 1992; Rhodius and Busby, 1998).

The role of ToxR may be reflected in the fact that, at wild-type levels of expression, ToxR interacts with the toxT promoter more readily than does TcpP (Fig. 4). Based on this observation, we propose a model in which TcpP interacts weakly with its promoter binding site, and ToxR serves to recruit TcpP or stabilize the poor promoter interaction of TcpP (Fig. 10). This is consistent with the observation that, at high concentrations, TcpP (a relatively inefficient promoter-binding protein) can activate transcription in the absence of ToxR (Fig. 3B). ToxR may enhance the activity of TcpP by a direct protein-protein interaction. Although ToxR-TcpP interaction has not been shown directly, we did observe that membranes containing ToxR and TcpP failed to bind to the -73 tox $T$ promoter deletion construct, whereas those containing TcpP alone bound well (Fig. 5, lanes 23 and 27). This may indicate that ToxR and TcpP interact away from the

(c) 2000 Blackwell Science Ltd, Molecular Microbiology, 38, 67-84 


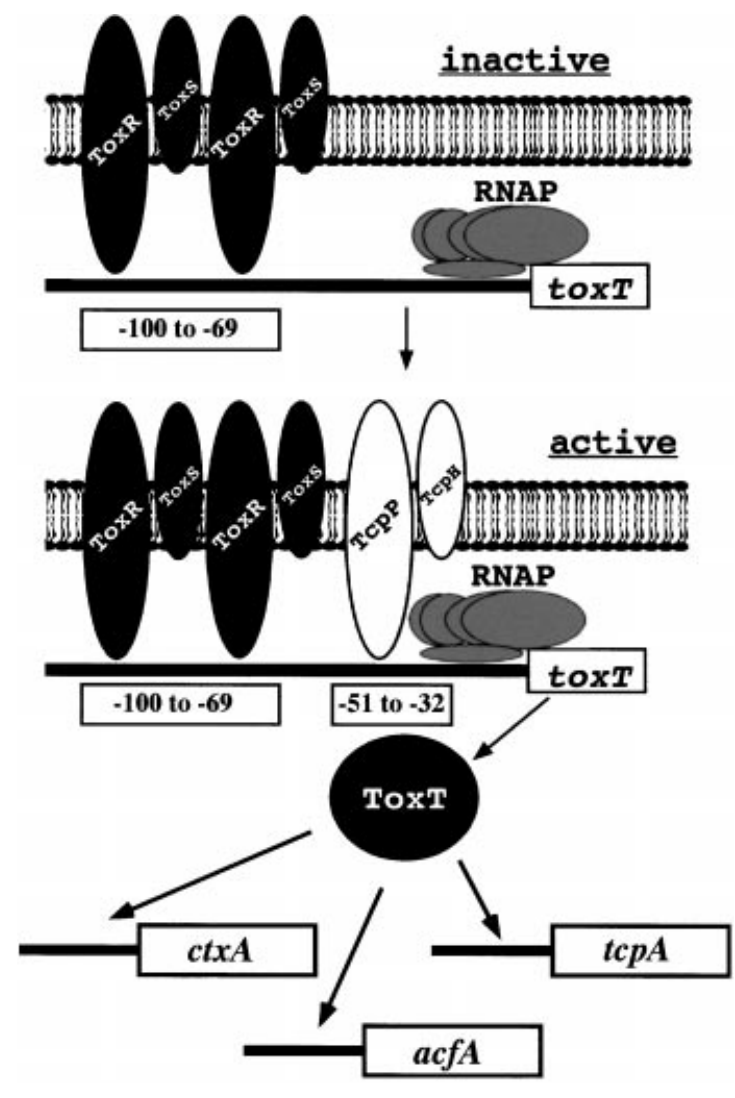

Fig. 10. Model for toxT promoter activation. Both ToxR and TcpP are required for the activation of the toxT promoter when these proteins are expressed at physiological levels. ToxR is produced constitutively in $V$. cholerae and binds the tox $T$ promoter from -100 to -69 . TcpP production is environmentally regulated. Once synthesized, TcpP binds to promoter sequences between -51 and -32 . As ToxR interacts more readily with the toxT promoter, it may serve to recruit TcpP to a relatively weak binding site, thus allowing contact with RNAP and initiation of transcription. Both ToxR and TcpP require accessory proteins (ToxS and $\mathrm{TcpH}$ respectively) for maximal activity. ToxR also protects a larger region of the toxT promoter from DNase I digestion than TcpP and may thus bind to multiple sites upstream of TcpP.

promoter, and deletion of the ToxR binding site from the tox $T$ promoter results in sequestration of TcpP away from its potential promoter site. In the case of the DNase I footprinting results (Fig. 6), in which the ToxR binding site is intact, but TcpP fails to protect its binding site in the presence of ToxR, the ToxR-TcpP association may alter the conformation of TcpP, such that it is competent for transcriptional activation, but less able to bind DNA. The ability of ToxR to stabilize partially a particular TcpP derivative (TcpP-H93L; Fig. $8 \mathrm{C}$ ) also suggests that the proteins may interact.

The ratio of TcpP to ToxR also appears to be critical for toxT activation, as overexpression of ToxRS in wildtype $V$. cholerae (O395) leads to a marked reduction in the amount of toxin produced $\left(404 \mathrm{ng} \mathrm{ml}^{-1} / \mathrm{OD}_{600}\right.$ in the wild type versus $14.8 \mathrm{ng} \mathrm{ml}^{-1} / \mathrm{OD}_{600}$ with ToxRS overexpression). This reduction is also consistent with an interaction between ToxR and $\mathrm{TcpP}$, and could result from titration of TcpP away from the tox $T$ promoter by excess unbound ToxR. Alternatively, it may represent occlusion of the TcpP binding site by ToxR. Plasmidencoded tox $R$ complements the $\Delta$ tox $R$ mutant used in this study (EK307) to a higher degree at low levels of induction (10 $\mu \mathrm{M}$ IPTG) than at higher levels $(1 \mathrm{mM})$, also suggesting that overexpression of ToxR is detrimental to toxT activation (E. S. Krukonis and A. Crawford, unpublished observations).

A number of tcp $P$ and tox $R$ point mutants were generated with the aim of isolating some defective for DNA binding or transcriptional activation activity. According to the hypothesis that ToxR recruits TcpP to the toxT promoter via a protein-protein interaction, TcpP DNAbinding mutants would be rescued for activation of toxT by being co-expressed with ToxR. In this scenario, ToxR would provide the promoter recognition function of the activation system, whereas TcpP would contact RNAP directly. One TcpP derivative tested, TcpP-H93L, behaves precisely as predicted from this model. When overexpressed alone in $V$. cholerae, TcpP-H93L cannot direct the expression of cholera toxin or TcpA; however, when co-expressed with endogenous levels of ToxR, this TcpP variant directs the expression of near wild-type levels of these two ToxT-dependent products (Fig. 8A and B). As TcpP-H93L is defective for DNA binding (Fig. 8D), ToxR appears to provide promoter recognition for toxT activation. Activation of the toxT promoter by TcpP-H93L in the presence of ToxR does not reach wild-type levels (Fig. $8 \mathrm{C}$ ), although toxin and TcpA production does. This may indicate that, after a certain cellular concentration of ToxT is achieved, further toxT activation does not lead to increased toxin and TcpA production. Our understanding of the mechanism by which ToxR rescues toxT activation by TcpP-H93L is complicated by the fact that ToxR partially stabilizes the relatively unstable TcpPH93L protein (Fig. $8 \mathrm{C}$ ); however, ToxR is clearly able to facilitate transcription activation activity by a TcpP mutant whose ability to bind DNA has been severely diminished. This suggests that ToxR may enhance the ability of TcpP to interact with the toxT promoter, thus positioning TcpP in close proximity to RNAP and leading to transcriptional activation. The fact that ToxR partially stabilizes TcpP$\mathrm{H} 93 \mathrm{~L}$ also suggests that these proteins may interact.

Two other interesting tcp $P$ mutants are TcpP-W68L and TcpP-R86A, both of which fail to activate transcription even in the presence of ToxR (Fig. $8 A-C$ ). In addition, both mutants fail to bind the toxT promoter. Why TcpPH93L, but not TcpP-W68L or TcpP-R86A, can be partially rescued for toxT activation by ToxR is unclear, but the latter two mutants may affect DNA binding as well as ToxR-TcpP interaction or the ability of TcpP to recognize 
Table 1. Strains, plasmids and oligonucleotides.

\begin{tabular}{|c|c|c|}
\hline Name & Description & Reference/source \\
\hline \multicolumn{3}{|l|}{ Strain } \\
\hline \multicolumn{3}{|l|}{ V. cholerae } \\
\hline O395 & Wild type, $\operatorname{Str}^{R}$ & Laboratory collection \\
\hline EK307 & O395 $\Delta$ toxR & This study \\
\hline RY1 & O395 $\Delta t c p P$ & Yu and DiRita (1999) \\
\hline EK459 & O395 $\Delta$ toxR $\Delta t c p P$ & This study \\
\hline EK385 & O395 lacZ::ompU-lacZ & This study \\
\hline EK410 & O395 $\Delta$ toxR lacZ::ompU-lacZ & \\
\hline EK739 & O395 toxR-G80S lacZ::ompU-lacZ & This study \\
\hline EK698 & O395 toxR-G80S & This study \\
\hline \multicolumn{3}{|l|}{ E. coli } \\
\hline $\mathrm{AC} 174$ & $\begin{array}{l}\text { IN (rrnD-rrnE)1 } \Delta \text { (lac)X74 rpsL galK2 recD1903::Tn 10d-Tet trpDC700::putPA1303 } \\
::[K a n S-C a m R-l a c] ~[K a n R-p o m p U-l a c Z]\end{array}$ & Crawford et al. (1998) \\
\hline $\mathrm{BMH} 71-18$ & thi supE $\Delta$ (lac-proAB) mutS::Tn 10 (Tet) & Promega \\
\hline $\mathrm{DH} 5 \alpha$ & supE44 $\Delta$ lacU169(F80lacZDM15) hsdR17 recA1 endA1 gyrA96 thi-1 relA1 & Laboratory collection \\
\hline DH92 & $\begin{array}{l}\text { IN (rrnD-rrnE)1 } \Delta \text { (lac)X74 rpsL galK2 recD1903::Tn 10d-Tet trpDC700::putPA1303 } \\
::[\text { KanS-CamR-lac] [KanR-ptoxT-lacZ] }\end{array}$ & Higgins and DiRita (1994) \\
\hline SM10גpir & thi-1 thr leu tonA lacY supE recA::RP4-2-Tc::Mu Km ${ }^{R}(\lambda$ pir) & Laboratory collection \\
\hline MM294 & $F^{-}$endA1 hsdR17 $\left(r_{K}^{-} m_{K}^{-}\right)$supE44 thi-1 relA1 rfbD1 spoT1 pRK2013 & Laboratory collection \\
\hline VJ787 & $\begin{array}{l}\text { IN (rrnD-rrnE)1 } 4 \text { (lac)X74 rpsL galK2 recD1903::Tn 10d-Tet trpDC700::putPA1303 } \\
::[\text { KanS-CamR-lac] [KanR-pctxA-lacZ] }\end{array}$ & $\begin{array}{l}\text { Bock and V. J. DiRita } \\
\text { (unpublished) }\end{array}$ \\
\hline \multicolumn{3}{|c|}{ \% } \\
\hline pACYC184 & Expression vector & Laboratory collection \\
\hline pVJ21 & pACYC184-toxRS & DiRita et al. (1991) \\
\hline pMMB66EH & Expression vector & Fürste et al. (1986) \\
\hline pMMB207 & Expression vector & Morales et al. (1991) \\
\hline pEK18 & p6891MCS-ompU-lacZ & This study \\
\hline pEK25 & pMMB66EH-tcpPH Amp ${ }^{R}$ & This study \\
\hline pEK32 & pMMB207-tcpPH Cm ${ }^{\mathrm{R}}$ & This study \\
\hline pEK38 & pMMB66EH-tcpP wt & This study \\
\hline pEK38-W68L & pMMB66EH-tcpP-W68L & This study \\
\hline pEK38-S77A & pMMB66EH-tcpP-S77A & This study \\
\hline pEK38-T85A & pMMB66EH-tcpP-T85A & This study \\
\hline pEK38-R86A & pMMB66EH-tcpP-R86A & This study \\
\hline pEK38-H93L & pMMB66EH-tcpP-H93L & This study \\
\hline pAlter & Mutagenesis vector & Promega \\
\hline pTL61T & Promoterless lac $Z$ vector & Linn and Pierre (1990) \\
\hline pTLI2 & pTL61T-ptoxT $(-172$ to +45$)$ & Higgins and DiRita (1994) \\
\hline pKAS32 & Suicide plasmid & Skorupski and Taylor (1996) \\
\hline pKAS32- $\Delta$ toxR & tox $R$ deletion suicide plasmid & This study \\
\hline pKAS32- $\Delta t c p P$ & tcpP deletion suicide plasmid & Yu and DiRita (1999) \\
\hline pKAS32-toxR-G80S & tox $R$ allelic exchange suicide plasmid & This study \\
\hline pSK-tcpP-HSV & HSV epitope-tagged tcpP in pBluescript & This study \\
\hline pEK41 & pMMB207-tcpP-HSV & This study \\
\hline pEK41-W68L & pMMB207-tcpP-W68L-HSV & This study \\
\hline pEK41-S77A & pMMB207-tcpP-S77A-HSV & This study \\
\hline pEK41-T85A & pMMB207-tcpP-T85A-HSV & This study \\
\hline pEK41-R86A & pMMB207-tcpP-R86A-HSV & This study \\
\hline pEK41-H93L & pMMB207-tcpP-H93L-HSV & This study \\
\hline \multicolumn{3}{|l|}{ Oligonucleotides } \\
\hline toxR $5^{\prime}$ BamHI & 5'-GGGGATCCATTGAGACTCAATGGAATTACCTTGATG-3' & This study \\
\hline toxRS $3^{\prime}$ BamHI & 5'-GGGGATCCCAATTAAATCACGCACTCTTTGCTTCA-3' & This study \\
\hline$\Delta$ toxR SOE top & 5'-CTCAAAAGAGATATCGATGCAAAATAGACACATC-3' & This study \\
\hline$\Delta$ toxR SOE bottom & 5'-GATGTGTCTATTTTGCATCGATATCTCTTTTGAG-3' & This study \\
\hline tcpP W68L & 5'-CAAATAATTAAAGCACTTTTGCCTCATGGATTTATC-3' & This study \\
\hline tcpP S77A & 5'-GGATTTATCAGCTCTGAAGCTCTAACTCAGGCAATC-3' & This study \\
\hline tcpP T85A & 5'-CAGGCAATCAAAAGAGCTCGTGATTTTTTTGAATG-3' & This study \\
\hline tcpP R86A & 5'-GGCAATCAAAAGAACCGCGGATTTTTTTGAATGATG-3' & This study \\
\hline tcp $P \mathrm{H} 93 \mathrm{~L}$ & 5'-GATTTTTTTGAATGATGAACTTAAGACGTTGATCG-3' & This study \\
\hline toxR G80S & 5'-GTTTGGCGAGAGCAAAGTTTTGAAGTCGATG-3' & This study \\
\hline $\operatorname{tcp} P 5^{\prime}$ & 5'-GGGGTACCGATAACTTTGCAACCGTT-3' & Yu and DiRita (1999) \\
\hline $\operatorname{tcp} P 3^{\prime}$ & 5'-TCCCCGCGGGACGATCTCAATACAACT-3' & Yu and DiRita (1999) \\
\hline$o m p U-l a c Z 5^{\prime}$ & 5'-CCCCGAATTCCCTAAATCGGGTCG-3' & Crawford et al. (1998) \\
\hline$o m p U-l a c Z 3^{\prime}$ & 5'-CCCCGAATTCACAGGAGCACGATC-3' & This study \\
\hline tcpP 5'EcoRI & 5'-GGGAATTCGTAAAGTAATGGGGTATG-3' & This study \\
\hline tcpP $3^{\prime} \mathrm{Xhol}$ & 5'-CCTGTAGCGCTCGAGTTAATTTTTTTGTGCATTC-3' & This study \\
\hline tcpH $3^{\prime}$ BamHI & 5'-GGGGATCCTACTCGTCACAGACT-3' & This study \\
\hline
\end{tabular}


Table 1. continued

\begin{tabular}{lll}
\hline Name & Description & Reference/source \\
\hline tcpP Smal-HSV & 5'-GGGGCATTTTTTGTGCATTCTAATGTC-3' $^{\prime}$ & This study \\
toxR 5' G80S & 5'-GACTTTGTTTGGCGAGAGCAAA-3' $^{\prime}$ & This study \\
toxR 5' G80wt & $5^{\prime}$-GACTTTGTTTGGCGAGAGCAAG-3' & This study \\
lacZ 5' & $5^{\prime}$-GATCTGAAGTCATCCGTAATC-3' & This study \\
lacZ 3' & 5'-TTAAGGCTCTCTGGCTTATTG-3' $^{\prime}$ & This study \\
\hline
\end{tabular}

a ToxR-induced toxT promoter architectural change. It should be noted that TcpP-R86A is somewhat unstable based on the detection of an HSV epitope-tagged version by Western blot (Fig. $8 \mathrm{C}$ ). However, TcpP-H93L is even more unstable than TcpP-R86A (Fig. $8 \mathrm{C}$ ), and its reduced protein levels are sufficient to activate toxT in the presence of ToxR. Thus, if TcpP-R86A had significant ToxRdependent toxT activation activity, it should have been observed.

Finally, a derivative of ToxR, ToxR-G80S, was defective for $o m p U$ activation, but maintained wild-type levels of tox $T-l a c Z$ activation and toxin production (Fig. 9). This allows us to differentiate between the role that ToxR plays on promoters such as ompU, in which it is the de facto activator, from its role on the toxT promoter, in which it serves to enhance the ability of TcpP to activate transcription. The fact that the transcriptional activation activity of ToxR is dispensable for toxT activation is consistent with the role of ToxR as an enhancer of TcpP function and not as an activator in the usual sense of the term.

Taken together, the data presented here clearly indicate a role for both TcpP and ToxR in activation of the toxT promoter. In our current model, TcpP binds the tox $T$ promoter at a polymerase-proximal site encompassing nucleotides -51 to -32 . ToxR occupies a more distal binding site from -100 to -69 , possibly involving more than one ToxR molecule (DiRita and Mekalanos, 1991; Harlocker et al., 1995). We propose that the role of ToxR in activation is to recruit the weaker binding TcpP to the $\mathrm{TcpP}$ promoter binding site, thereby situating TcpP in close enough proximity to RNAP to initiate transcription (Fig. 10). Whether this recruitment occurs by direct protein-protein interaction or alteration of the toxT promoter DNA to allow TcpP access to a position that is competent for transcriptional activation remains to be determined, as does the nature of the putative interaction of TcpP with RNAP. As ToxR appears to be produced under various environmental conditions in $V$. cholerae (DiRita et al., 1996), whereas TcpP is transcriptionally regulated in response to both temperature and $\mathrm{pH}$ (Thomas et al., 1995; Carroll et al., 1997; Häse and Mekalanos, 1998; Skorupski and Taylor, 1999), we hypothesize that ToxR constitutively binds the tox $T$ promoter, but activation does not ensue until TcpP is synthesized. At that point, ToxR directs TcpP to its position on the toxT promoter to contact RNAP and activate transcription (Fig. 10). Thus, the V. cholerae virulence cascade is set in motion.

\section{Experimental procedures}

\section{Bacterial strains and plasmids}

All E. coli and $V$. cholerae strains, plasmids and oligonucleotides used in this study are listed in Table 1. The construction of E. coli strain DH92 harbouring a toxT-lacZ fusion on the chromosome has been described previously (Higgins and DiRita, 1994). Strains were grown in LB medium at $30^{\circ} \mathrm{C}$ or $37^{\circ} \mathrm{C}$. Strains were maintained in LB medium containing $20 \%$ glycerol at $-70^{\circ} \mathrm{C}$. Antibiotics were used at the following concentrations: streptomycin $100 \mu \mathrm{g} \mathrm{ml}^{-1}$; ampicillin $100 \mu \mathrm{g}$ $\mathrm{ml}^{-1}$; tetracycline $12.5 \mu \mathrm{g} \mathrm{ml}^{-1}$; chloramphenicol $25 \mu \mathrm{g} \mathrm{ml}^{-1}$; and kanamycin $40 \mu \mathrm{g} \mathrm{ml}^{-1}$. DNA was transferred into $V$. cholerae by electroporation $(2.2 \mathrm{kV}, 200 \mathrm{~W})$ using an E. coli Pulsor (Bio-Rad) or by conjugation using an E. coli strain harbouring the mobilization plasmid pRK2013. E. coli strains were transformed by standard methods (Sambrook et al., 1989).

\section{Construction of $\Delta$ toxR, $\Delta \mathrm{tcpP}$ and $\Delta$ toxR $\Delta \mathrm{tcpP}$ strains}

The $\Delta$ toxR strain EK307 was constructed using the suicide vector pKAS32 (Skorupski and Taylor, 1996), into which a DNA fragment obtained by SOEing polymerase chain reaction (PCR) (Higuchi, 1990) containing 233 nucleotides upstream of the toxR start codon fused to the start codon of toxS and 805 downstream nucleotides had been inserted. This tox $R$ deletion fragment was generated by first amplifying the $\mathrm{N}$-terminal end of toxR up to the start codon and engineering a $5^{\prime} \mathrm{BamHI}$ site using primers tox $R 5^{\prime} \mathrm{BamHI}$ and $\Delta$ toxR SOE bottom (Table 1). Next, the toxS and downstream sequences were amplified, and a $3^{\prime} B a m H I$ site was engineered using oligos toxRS $3^{\prime} B a m H I$ and $\triangle$ toxR SOE top. Oligos $\Delta$ tox $R$ SOE bottom and $\Delta$ tox $R$ SOE top overlap with one another and encode the fusion junction. After the separate PCR reactions, the products were cleaned with Qiex beads (Qiagen), and a final PCR reaction was performed using the primers toxR $5^{\prime} B a m H I$ and toxRS $3^{\prime} B a m H I$ to generate a fragment with the internal tox $R$ deletion. This product was digested with $B a m H I$, inserted into Bgll-cut pKAS32 and mated into $V$. cholerae strain 0395 using the E. coli strain SM10入pir by filter conjugation. The $\triangle t c p P$ strain RY1 was constructed similarly as described by Yu and DiRita (1999). The $\Delta$ toxR $\Delta$ tcpP strain EK459 was constructed by mating strain EK307 ( $\Delta$ toxR) with SM10入pir 
harbouring the pKAS32- $\Delta$ tcpP plasmid. Recombination and loss of the wild-type allele was confirmed by PCR analysis using primers toxR $5^{\prime} B a m \mathrm{HI} /$ toxRS $3^{\prime} B a m H I$ (toxR) or tcpP $5^{\prime} / t c p P 3^{\prime}$ (tcpP). PCR reactions for cloning were performed with the Expand High Fidelity PCR system (Boehringer Mannheim).

\section{DNA gel mobility shift assays}

Membrane extracts derived from the various $V$. cholerae strains were prepared as described previously (Miller et al., 1987) and stored at $-70^{\circ} \mathrm{C}$ in $25 \%$ sucrose with $5 \mathrm{mM}$ EDTA. Protein concentrations were determined using the Bio-Rad protein quantification assay. tox $T$ promoter fragments were end labelled by digesting $\approx 10-20 \mu \mathrm{g}$ of the plasmid pTLI2 (or appropriate deletion derivatives) with Sall in a $30 \mu \mathrm{l}$ volume, followed by a fill-in reaction using 5 units of Klenow DNA polymerase (New England Biolabs), 2 mM dGTP, dATP and dTTP as well as $30 \mu \mathrm{Ci}$ of $\left[\alpha^{-32} \mathrm{P}\right]-\mathrm{dCTP}$ (Amersham). After a $15 \mathrm{~min}, 70^{\circ} \mathrm{C}$ heat inactivation of the Klenow enzyme, the promoter fragment was liberated by a final digestion with $B a m H I$. The promoter fragment was then purified by PAGE. Probe was eluted from the gel overnight at $37^{\circ} \mathrm{C}$ and adjusted to a concentration of 20000 c.p.m. $\mu l^{-1}$. Various concentrations of membrane were then mixed with 3000 c.p.m. or 20000 c.p.m. of probe in the presence of $10 \mu \mathrm{g} \mathrm{ml}^{-1}$ sheared salmon sperm DNA and $10 \mathrm{mM}$ Tris, $\mathrm{pH} 7.4$, $1 \mathrm{mM}$ EDTA, $5 \mathrm{mM} \mathrm{NaCl}, 50 \mathrm{mM} \mathrm{KCl}, 50 \mu \mathrm{g} \mathrm{ml}^{-1}$ BSA in a $20 \mu \mathrm{l}$ final volume. Binding reactions were performed at $30^{\circ} \mathrm{C}$ for $30 \mathrm{~min}$, and samples were run on a $6 \%$ polyacrylamide non-denaturing gel that had been prerun with $20 \mu \mathrm{l}$ of $5 \%$ thioglycolic acid in each well to prevent membrane-DNA complexes from entering the gel. DNA binding was determined by comparing the amount of free probe and the amount of probe retained in the well. In the case of epitope-tagged TcpP derivatives, Western blots were used to determine the amount of TcpP in each membrane preparation from the EK459 V. cholerae background. Membrane concentrations were adjusted to give equivalent levels of TcpP in the binding reactions. For TcpP-wt, TcpP-W68L, TcpP-S77A and TcpP-T85A, $2 \mathrm{mg} \mathrm{ml}^{-1}$ and $5 \mathrm{mg} \mathrm{ml}^{-1}$ membranes were used. For EK459 + pMMB207 (empty vector), TcpP-R86A and TcpP-H93L, $4 \mathrm{mg} \mathrm{m}^{-1}$ and $10 \mathrm{mg}$ $\mathrm{ml}^{-1}$ were used. In order to have equivalent amounts of total membrane proteins in the binding reactions, an additional $2 \mathrm{mg} \mathrm{ml}^{-1}$ and $5 \mathrm{mg} \mathrm{ml}^{-1}$ EK459 + pMMB207 membranes were added to the first four reactions to achieve a final concentration of $4 \mathrm{mg} \mathrm{ml}^{-1}$ and $10 \mathrm{mg} \mathrm{ml}^{-1}$ total protein. Western blots were then used to show the relative levels of TcpP-HSV present in each binding reaction.

\section{DNase I footprinting analysis}

DNase I footprinting reactions were performed as described previously (Crawford et al., 1998) with the exception that $20 \mathrm{mg} \mathrm{ml}^{-1} \mathrm{~V}$. cholerae membranes were used rather than E. coli. Reactions were digested for $2 \mathrm{~min}$ at room temperature using 0.1 units of DNase I. toxT promoter fragments were cut from the vector pTLI2 with either Sall (bottom strand labelling) or BamHI (top strand labelling) first, filled in and then cut with BamHI or Sall, respectively, to generate differentially labelled strands. Labelled probe (70 000 c.p.m.) was used in each $70 \mu$ l reaction.

\section{Construction of tcpP expression plasmids}

The tcpP gene or tcpPH operon was amplified from chromosomal DNA derived from $\mathrm{O} 395$ (Classical) or E7946 (El Tor) V. cholerae strains using the Expand High Fidelity PCR system (Boehringer Mannheim) and the oligonucleotides tcpP $5^{\prime} E c o R l / t c p P \quad 3^{\prime} X$ hol or tcpP $5^{\prime} E c o R l / t c p H$ $3^{\prime}$ BamHI. After PCR amplification, products were digested with EcoRI-Xhol or EcoRI-BamHI and ligated into EcoRISall or EcoRI-BamHI cut pMMB66EH. TcpP $(\mathrm{H})$ inserts were subsequently moved to pMMB207 as EcoRI-Pstl or EcoRIBamHI fragments to allow inducible expression in a chloramphenicol-resistant vector. pMMB66EH and pMMB207 were kindly supplied by Michael Bagdasarian (Michigan State University).

\section{Construction of tcpP and toxR site-directed mutants}

Site-directed mutants in tcp $P$ were generated by cloning the tcpP gene into the mutagenesis vector pAlter (Promega), annealing oligonucleotides containing the changes of interest to the wild-type sequence and carrying out a polymerization and ligation reaction according to the manufacturer's instructions. Mutants were confirmed by DNA sequencing (Sequenase; US Biochemicals). Mutant alleles were then liberated from pAlter as an EcoRI-Pstl fragment and cloned into an EcoRI-Pstl-digested expression vector pMMB66EH (Fürste et al., 1986). Mutant alleles were mated into $V$. cholerae derivatives and used to measure complementation of a chromosomal $\Delta t c p P$ allele. The toxR-G80S allele was constructed similarly in pAlter. After sequencing, toxR-G80S was liberated as a BamHI fragment and ligated into Bgll-digested suicide plasmid pKAS32. The resulting pKAS-toxR-G80S was transformed into E. coli SM10גpir and then mated into O395 or EK385 (O395 lacZ::ompU-lacZ) by filter conjugation. Replacement of the wild-type tox $R$ locus was confirmed by selective PCR using $5^{\prime}$ oligonucleotides with a $3^{\prime}$ end corresponding to the site of the G80S mutation ( $5^{\prime}$ toxR-G80S or $5^{\prime}$ toxR-G80wt) and the $3^{\prime}$ oligo toxRS $3^{\prime} B a m H I$ with annealing at $55^{\circ} \mathrm{C}$. A carboxy-terminal HSV epitope tag was added to various $t c p P$ alleles by amplifying the wild-type $t c p P$ allele by PCR using primers tcp $P 5^{\prime} E c o R I$ and tcp $P 3^{\prime} S m a l$, which remove the TAA stop codon and provide a portion of a Smal site. This PCR product was treated with mung bean nuclease (New England Biolabs) to ensure blunt ends, cut with $\mathrm{EcoRI}$ and ligated into EcoRI-Smal-digested pMMB66EH. The resulting plasmid was digested with Smal, and an HSV epitope-tagging oligo was inserted by ligation (Novagen). The wild-type HSV-tagged tcpP allele was moved into pBluescript SK (Stratagene) as an EcoRl-Pstl fragment, and then various tcp $P$ mutant alleles were ligated into this backbone as EcoRI-BstEll cassettes, replacing the $5^{\prime}$ end of the wildtype tcpP gene. The various tcpP mutants were then shuttled as EcoRI-Pstl fragments into the inducible expression plasmid pMMB207. Expression was assessed after growth in $1 \mathrm{mM}$ IPTG for various times by Western blotting using an 
anti-HSV monoclonal antibody (Novagen) followed by an anti-mouse $2^{\circ}$ antibody conjugated to alkaline phosphatase (AP; Gibco BRL).

\section{Measurement of toxT-lacZ, ompU-lacZ or ctxA-lacZ activation}

Cells were grown overnight at $37^{\circ} \mathrm{C}\left(E\right.$. coli) or $30^{\circ} \mathrm{C}(\mathrm{V}$. cholerae) in LB, diluted 1:100 in fresh LB with $1 \mathrm{mM}$ IPTG, where appropriate, and grown for an additional $3 \mathrm{~h}$ at $37^{\circ} \mathrm{C}$ (E. coll) or $4 \mathrm{~h}$ at $30^{\circ} \mathrm{C}$ (V. cholerae). Samples of 20-100 $\mu \mathrm{l}$ of culture were used for measurement of $\beta$-galactosidase activity as described previously (Miller, 1972), and the $\mathrm{OD}_{600}$ was determined and used to normalize cultures for subsequent Western blot analysis of ToxR or TcpA expression and calculation of cholera toxin expression.

\section{Measurement of cholera toxin production}

Cholera toxin levels of various derivatives were determined by growing cultures overnight at $30^{\circ} \mathrm{C}$ in LB with $1 \mathrm{mM}$ IPTG where appropriate. The culture $(1 \mathrm{ml})$ was microcentrifuged for $5 \mathrm{~min}$, and the resulting supernatant was diluted in a $\mathrm{GM}_{-1}$ ganglioside-coated microtitre plate as described previously (Svennerholm and Holmgren, 1978). Toxin binding was revealed with polyclonal anti-CtxB antibodies provided by Dr Michael Bagdasarian (Michigan State University) at a 1:1000 dilution, followed by an anti-rabbit AP-conjugated $2^{\circ}$ antibody at 1:2000 dilution (Gibco BRL). The chromagenic substrate ONPG (Sigma) was used at $4 \mathrm{mg} \mathrm{ml}^{-1}$, and Abs405 was measured using an enzyme-linked immunosorbent assay (ELISA) plate reader.

\section{Measurement of TcpA and ToxR production}

V. cholerae cells were grown overnight in LB at $30^{\circ} \mathrm{C}$ with $1 \mathrm{mM}$ IPTG if appropriate. Cells $(1 \mathrm{ml})$ were microcentrifuged and resuspended in 200-500 $\mathrm{ml}$ of sample buffer (Sambrook et al., 1989). Cells were resuspended in appropriate volumes to normalize for different $\mathrm{OD}_{600}$ readings. Cells were boiled for $5 \mathrm{~min}$, and equal volumes of cell extract were loaded for SDS-PAGE analysis. TcpA was detected by Western blot analysis using anti-TcpA polyclonal antibodies generously provided by Dr Ron Taylor (Dartmouth Medical School) at 1:100 000 dilution, and ToxR was detected using polyclonal anti-ToxR antibodies kindly supplied by Dr John Mekalanos (Harvard Medical School) at 1:1000 dilution.

\section{Detection of OmpU or OmpT protein}

$V$. cholerae cultures were grown for various times, then $1 \mathrm{ml}$ of culture was pelleted and resuspended in $1 \times$ sample buffer (Sambrook et al., 1989). The volume of resuspension was adjusted to normalize for culture $\mathrm{OD}_{600}$. Whole-cell pellets were boiled for $5 \mathrm{~min}$ and run on a $12 \%$ polyacrylamide gel at $150 \mathrm{~V}$, followed by staining with Coomassie brilliant blue. OmpU (40 kDa) and OmpT (38 kDa) are predominant bands in the whole-cell lysates.

\section{Acknowledgements}

We would like to thank Adam Crawford, Stephen Cendrowski, Claudia Häse and Ana Coehlo for many helpful discussions of this work. This work was supported by grants Al31645 and Al45125 to V.J.D. E.S.K was supported by NRSA grant Al09942. DNA sequence analysis was supported in part by grant M01-RR 00042 to the University of Michigan Clinical Research Center.

\section{References}

Carroll, P.A., Tashima, K.T., Rogers, M.B., DiRita, V.J., and Calderwood, S.B. (1997) Phase variation in tcpH modulates expression of the ToxR regulon in Vibrio cholerae. Mol Microbiol 25: 1099-1111.

Chakrabarti, S.R., Chaudhuri, K., Sen, K., and Das, J. (1996) Porins of Vibrio cholerae: purification and characterization of OmpU. J Bacteriol 178: 524-530.

Champion, G.A., Neely, M.N., Brennan, M.A., and DiRita, V.J. (1997) A branch in the ToxR regulatory cascade of Vibrio cholerae revealed by characterization of toxT mutant strains. Mol Microbiol 23: 323-331.

Collado-Vides, J., Magasanik, B., and Gralla, J.D. (1991) Control site location and transcriptional regulation in Escherichia coli. Microbiol Rev 55: 371-394.

Crawford, J.A., Kaper, J.B., and DiRita, V.J. (1998) Analysis of ToxR-dependent transcription activation of $o m p U$, the gene encoding a major envelope protein in Vibrio cholerae. Mol Microbiol 29: 235-246.

DiRita, V.J., and Mekalanos, J.J. (1991) Periplasmic interaction between two membrane regulatory proteins, ToxR and ToxS, results in signal transduction and transcriptional activation. Cell 64: 29-37.

DiRita, V.J., Parsot, C., Jander, G., and Mekalanos, J.J. (1991) Regulatory cascade controls virulence in Vibrio cholerae. Proc Natl Acad Sci USA 88: 5403-5407.

DiRita, V.J., Neely, M., Taylor, R.K., and Bruss, P.M. (1996) Differential expression of the ToxR regulon in classical and El Tor biotypes of Vibrio cholerae is due to biotype-specific control over toxT expression. Proc Natl Acad Sci USA 93: 7991-7995.

Elliott, T. (1992) A method for constructing single-copy lac fusions in Salmonella typhimurium and its application to the hemA-prfA operon. J Bacteriol 174: 245-253.

Fürste, J.P., Pansegrau, W., Frank, R., Blöcker, H., Scholz, P., Bagdasarian, M., et al. (1986) Molecular cloning of the plasmid RP4 primase region in a multi-host-range tac $P$ expression vector. Gene 48: 119-131.

Harlocker, S.L., Bergstrom, L., and Inouye, M. (1995) Tandem binding of six OmpR proteins to the ompF upstream regulatory sequence of Escherichia coli. $J$ Biol Chem 270: 26849-26856.

Häse, C.C., and Mekalanos, J.J. (1998) TcpP protein is a positive regulator of virulence gene expression in Vibrio cholerae. Proc Natl Acad Sci USA 95: 730-734.

Higgins, D.E., and DiRita, V.J. (1994) Transcriptional control of toxT, a regulatory gene in the ToxR regulon of Vibrio cholerae. Mol Microbiol 14: 17-29.

Higgins, D.E., Nazareno, E., and DiRita, V.J. (1992) The virulence gene activator ToxT from Vibrio cholerae is a 
member of the AraC family of transcriptional activators. $J$ Bacteriol 174: 6974-6980.

Higuchi, R. (1990) Recombinant PCR. In PCR Protocols: a Guide to Methods and Applications. Innis, M.A., Gelfand, D.H., Sninsky, J.J., and White, T.J. (eds). San Diego: Academic Press, p. 177-183.

Ishihama, A. (1992) Role of RNA polymerase a subunit in transcription activation. Mol Microbiol 6: 3283-3288.

Karaolis, D.K., Johnson, J.A., Bailey, C.C., Boedeker, E.C., Kaper, J.B., and Reeves, P.R. (1998) A Vibrio cholerae pathogenicity island associated with epidemic and pandemic strains. Proc Natl Acad Sci USA 95: 3134-3139.

Karaolis, D.K.R., Somara, S., Manaeval, D.R., Jr, Johnson, J.A., and Kaper, J.B. (1999) A bacteriophage encoding a pathogenicity island, a type-IV pilus and a phage receptor in cholera bacteria. Nature 399: 375-379.

Kovach, M.E., Shaffer, M.D., and Peterson, K.M. (1996) A putative integrase gene defines the distal end of a large cluster of ToxR-regulated colonization genes in Vibrio cholerae. Microbiology 142: 2165-2174.

Kovacikova, G., and Skorupski, K. (1999) A Vibrio cholerae LysR homolog, AphB, cooperates with AphA at the tcpPH promoter to activate expression of the ToxR virulence cascade. J Bacteriol 181: 4250-4256.

Li, C.C., Crawford, J.A., DiRita, V.J., and Kaper, J.B. (2000) Molecular cloning and transcriptional regulation of ompT, a ToxR-repressed gene in Vibrio cholerae. Mol Microbiol 35: 189-203.

Makino, K., Amemura, M., Kawamoto, T., Kimura, S., Shinagawa, H., Nabata, A., et al. (1996) DNA binding of PhoB and its interaction with RNA polymerase. $J \mathrm{Mol} B i o l$ 259: 15-26.

Martínez-Hackert, E., and Stock, A.M. (1997a) Structural relationships in the OmpR family of winged-helix transcription factors. J Mol Biol 269: 301-312.

Martínez-Hackert, E., and Stock, A.M. (1997b) The DNAbinding domain of OmpR: crystal structure of a winged helix transcription factor. Structure 5: 109-124.

Miller, J.H. (1972) Experiments in Molecular Genetics. Cold Spring Harbor, NY: Cold Spring Harbor Laboratory Press.

Miller, V.L., and Mekalanos, J.J. (1988) A novel suicide vector and its use in construction of insertion mutations: osmoregulation of outer membrane proteins and virulence determinants in Vibrio cholerae requires toxR. $J$ Bacteriol 170: 2575-2583.

Miller, V.L., Taylor, R.K., and Mekalanos, J.J. (1987) Cholera toxin transcriptional activator ToxR is a transmembrane DNA binding protein. Cell 48: 271-279.

Murley, Y.M., Carroll, P.A., Skorupski, K., Taylor, R.K., and Calderwood, S.B. (1999) Differential transcription of the tcpPH operon confers biotype-specific control of the Vibrio cholerae ToxR virulence regulon. Infect Immun 67: 51175123.

Ogierman, M.A., Voss, E., Meaney, C., Faast, R., Attridge,
S.R., and Manning, P.A. (1996) Comparison of the promoter proximal regions of the toxin-co-regulated tcp gene cluster in classical and El Tor strains of Vibrio cholerae 01. Gene 170: 9-16.

Ottemann, K.M., DiRita, V.J., and Mekalanos, J.J. (1992) ToxR proteins with substitutions in residues conserved with OmpR fail to activate transcription from the cholera toxin promoter. J Bacteriol 174: 6807-6814.

Peterson, K.M., and Mekalanos, J.J. (1988) Characterization of the Vibrio cholerae ToxR regulon: identification of novel genes involved in intestinal colonization. Infect Immun 56: 2822-2829.

Pratt, L.A., and Silhavy, T.J. (1994) OmpR mutants specifically defective for transcriptional activation. $J$ Mol Biol 243: 579-594.

Rhodius, V.A., and Busby, S.J.W. (1998) Positive activation of gene expression. Curr Opin Microbiol 1: 152-159.

Russo, F.D., Slauch, J.M., and Silhavy, T.J. (1993) Mutations that affect separate functions of OmpR the phosphorylated regulator of porin transcription in Escherichia coli. $J \mathrm{Mol}$ Biol 231: 261-273.

Sambrook, J., Fritsch, E.F., and Maniatis, T. (1989) Molecular Cloning: a Laboratory Manual, 2nd edn. Cold Spring Harbor, NY: Cold Spring Harbor Laboratory Press.

Skorupski, K., and Taylor, R.K. (1996) Broad-host-range positive selection vectors for allelic exchange. Gene 169: 47-52.

Skorupski, K., and Taylor, R.K. (1999) A new level in the Vibrio cholerae virulence cascade: aphA is required for transcriptional activation of the tcpPH operon. Mol Microbiol 31: 763-771.

Svennerholm, A.M., and Holmgren, J. (1978) Identification of the Escherichia coli heat-labile enterotoxin by means of a ganglioside immunosorbent assay $\left(\mathrm{GM}_{1}\right.$-ELISA) procedure. Curr Microbiol 1: 19-23.

Taylor, R.K., Miller, V.L., Furlong, D.B., and Mekalanos, J.J. (1987) Use of phoA gene fusions to identify a pilus colonization factor coordinately regulated with cholera toxin. Proc Natl Acad Sci USA 84: 2833-2837.

Thomas, S., Williams, S.G., and Manning, P.A. (1995) Regulation of tcp genes in classical and El Tor strains of Vibrio cholerae 01. Gene 166: 43-48.

Waldor, M.K., and Mekalanos, J.J. (1996) Lysogenic conversion by a filamentous phage encoding cholera toxin. Science 272: 1910-1914.

Wong, S.M., Carroll, P.A., Rahme, L.G., Ausubel, F.M., and Calderwood, S.B. (1998) Modulation of expression of the ToxR regulon in Vibrio cholerae by a member of the twocomponent family of response regulators. Infect Immun 66: 5854-5861.

Yu, R.R., and DiRita, V.J. (1999) Analysis of an autoregulatory loop controlling ToxT, cholera toxin, and toxincoregulated pilus production in Vibrio cholerae. J Bacteriol 181: 2584-2592. 